\title{
Ameliorative Role of Potassium on Water Relations, Antioxidative Enzymes and Yield of Sweet Corn Genotypes under Water Deficit
}

\author{
S. Rao ${ }^{1}$, S. Singh ${ }^{2}$ and N. Singh ${ }^{1^{*}}$ \\ Received: 27 $7^{\text {th }}$ May 2020 / Accepted: 01 ${ }^{\text {st }}$ November 2021
}

\begin{abstract}
Purpose: In recent years, it has been revealed that potassium delivers drought stress tolerance in many crops. This nutrient is not yet tested for sweetcorn. Therefore, this study investigated the impact of drought and soil applied potassium on water relations, antioxidant enzymes and yield attributes of two sweet corn genotypes in India.

Research Method: The factorial experiment was set-up with 5 replications in complete randomized block design. Factor one comprised of two genotypes (Sugar-75 and NSC 901B), factor two had six different water treatments and factor three with two different potassium levels. This investigation was designed with two genotypes of sweet corn to study the interaction between various water deficit conditions and soil applied potassium.
\end{abstract}

Findings: Drought stress significantly decreased the water potential, osmotic potentialand relative water content and caused osmotic stress in sweet corn plants. Antioxidant enzymes activities were found to be upregulated under drought stress while yield attributes were adversely affected by water deficit. Application of potassium increased the adaptation to water deficit stress by positively modulating the tested parameters. Results procured from this study suggested that exogenously applied potassium mitigated the drought mediated constrains on sweet corn and enhanced drought tolerance.

Research Limitations: The experiments were carried out in polythene pots to provide a uniform soil condition which was the main limitation when compared to field trials.

Originality/value: Results of this experiment revealed that exogenous application of potassium can alleviate the damaging effect of drought stress on sweet corn. However, more research on other crops is needed to ensure its long-term sustainability.

Keywords: Antioxidative enzymes, potassium, water deficit, water relations, yield attributes

\section{INTRODUCTION}

World population is increasing exponentially which is a major reason for increasing food demands. One in every nine persons and overall 820 million are suffering from serious hunger in the world (FAO, 2019). The goal of sustainable development programs is to ensure food safety for everyone till 2030 and is hindered by 820 million malnourished people (Richardson et al., 2018). Many abiotic stresses particularlywater deficit stress adversely affected the productivity of crops (Ashraf et al., 2018). Water deficit stress has a detrimental influence on crop because it suppresses the growth at distinct stages like root elongation, tiller expansion, anthesis, grain formation, dry matter partioning, harvest index and also influences overall development of plants

\begin{tabular}{l}
\hline Botany Department, IIHS Kurukshetra University, \\
Kurukshetra \\
${ }^{2}$ Botany Department, Govt. College for Girls Cheeka \\
${ }^{3 *}$ Botany Department, Kurukshetra University, \\
Kurukshetra \\
nsheorankuk@yahoo.com \\
(D) https://orcid.org/0000-0002-6690-3179
\end{tabular}


(Ashraf et al., 2017). Exogenous application of nutrients is one of the strategies which alleviate the efficacy of water deficit on the growth of crop plants (Ahmad et al., 2018). Potassium plays a significant role in various processes i.e. carbon assimilation, translocation, activation of many enzymes, respiration, plant water relation, metabolism, osmoregulation, development, growth and yield (Waraich et al., 2011). Potassium plays a significant role in the osmotic adjustment, opening and closing of stomata, activation of enzymes and is a part of the plant structure that optimizes multiple physiological and biochemical processes and eventually enhances plant growth and yield (Ahmad et al., 2018).

Among all the different types of maize, sweet corn (Zea mays var. saccharata) is the commercially used maize. It is a sugar rich maize variety and designed as a miracle crop. Sweet corn has high dietary benefits. Sweet corn has a high concentration of zeaxanthin, lutein and other carotenoids (Junpatiw et al., 2013). Sweet corn postpones aging since it is a powerhouse of antioxidants (Dewanto et al., 2002). It is categorized in six most popular vegetables in the United States of America (Ranum, 2014). Frozen sweet corn is a highly consumed product in the United States of America, after frozen tomatoes and potatoes (Lucier and Dettmann, 2008). The objective of this study was to investigate the impact of potassium application on water relation, antioxidative enzymes and yield parameters in sweetcorn plants under water deficit.

\section{MATERIALS AND METHODS}

This study was carried out for three consecutive years (2017-19) during spring season in net house of Botany Department, Kurukshetra University, Kurukshetra, Haryana, India. This research exploration was conducted by taking two genotypes of sweet corn (SUGAR-75 and NSC901B). Sweet corn seeds were sown during $2^{\text {nd }}$ week of March each year and water treatment was given after germination. The crop was raised in polythene bags having a $25 \mathrm{~cm}$. diameter and filled with $10 \mathrm{~kg}$ of soil (pH-7.70, EC(ds/m)- 0.51 , Organic Carbon (\%)- 0.5, Available nitrogen (ppm)- 60.0, Accessible phosphorous (ppm), Accessible potassium (ppm) and Available sulphur (ppm)-78.0).

Experimental analysis was laid out in completely randomized Designs (CRD).Statistical analysis was performed using three factors OP STAT developed by Sheoran et al., (1998).

Water deficit conditions were generated by withholding irrigation at different periods of time.

a) Fully irrigated (FI): After germination, everyday irrigation. (Control)

b) Fully drought (FD): After germination, no irrigation.

c) Moderate early drought (MED): After germination, weekly irrigation in every 7 days.

d) Severe early drought (SED): After germination irrigation in every 14 days.

e) Moderate late drought (MED): Full irrigation until 50 days, then irrigation in every 7 days.

f) Severe late drought (SED): Fully irrigation until 50 days, then irrigation in every 14 days.

After germination (10 days after sowing), three different potassium solutions with different concentrations $\left(0 \quad \mathrm{ppm}, \mathrm{K}_{1}=500 \mathrm{ppm}\right.$ and $\mathrm{K}_{2}=700 \mathrm{ppm}$ ) were applied in experimental units in the form of muriate of potash in addition to the existing level $(52 \mathrm{ppm})$ in the soil medium with recommended doses of nitrogen and phosphorous. Samples for different parameters were taken at vegetative, silking and post silking stages. Five replicas of every treatment alongwith control were examined each time to record the different parameters referenced underneath:

\section{Water relations}

The leaf water potential $\left(\Psi^{\mathrm{w}}\right)$ was measured using a pressure chamber (Model 3005, Soil Moisture Equipment Corporation). Took 3rd extended leaf at vegetative, silking and post silking stages and cut it with sharp edged cutter then placed 
it onto the lip of chamber. The pressure was recorded when sap or exudates simply oozed out. This pressure (-Mpa) was water potential of the respective tissue.

Leaf Osmotic potential $\left(\Psi^{\mathrm{S}}\right)$ of vegetative, silking and post silking stages was estimated by Psychrometric method (Model 5100-B Vapour pressure osmometer). Third fully expanded leaf from stem was withdrawn and put in tight stopper glass tubes. Tubes were warmed on heating bath intended for 1 hour in order to remove turgidity of tissues. Executed constituents were placed in deep refrigerator. Tissues were squashed by the help of glass rod with rounded finish. Filter paper disc was fully dipped in extricated sap of leaf and soaked disc was quickly kept in concave depression of holder. Readings displayed on osmometer were noted. Osmometer was adjusted by Osmotically Reference Standards of Sodium chloride (Wescor, Inc.USA) and alignment was done as below:

$$
\begin{aligned}
& 40 \text { osmo }=-1 \text { bar } \\
& -10 \text { bar }=-1 \mathrm{Mpa}
\end{aligned}
$$

Leaf Relative water content (RWC) of vegetative, silking and post silking stages was determined by the method of Weatherley (1950). Third completely expanded leaf, commencing from top of plant was taken and weigh quickly to conclude the fresh weight. Leaves were then floated on surface of water in clogged petri dishes for 3 hours in subtle light at consistent temperature of $25^{\circ} \mathrm{C} \pm 1^{\circ} \mathrm{C}$ and again weighed (fully turgid). After that the dry weight was recorded by keeping it in an oven at $85^{\circ} \mathrm{C}$ for 72 hours.

\section{Antioxidant enzymes}

Superoxide dismutase (SOD) activity was determined as per Giannepols and Ries (1977). 50 $\mathrm{mg}$ of leaves were crushed in a pestle and mortar with $2 \mathrm{ml}$ of $0.1 \mathrm{M}$ EDTA-phosphate buffer ( $\mathrm{pH}$ 7.8) and then final volume was increased to $100 \mathrm{ml}$ by adding double distilled water. Centrifugation was done at $15000 \mathrm{rpm}$ for 10 minutes and the crude extract was utilized as a resultant. Then, took $0.1 \mathrm{ml}$ from this crude extract and added 0.9 $\mathrm{ml}$ of double distilled water, followed by $0.5 \mathrm{ml}$ of $300 \mathrm{mM}, \mathrm{Na}_{2} \mathrm{CO}_{3}(\mathrm{pH} 10.2), 0.5 \mathrm{ml}$ of $378 \mu \mathrm{M}$ p-nitrobluetetrazolium chloride(NBT), $0.5 \mathrm{ml}$ of $78 \mathrm{mM}$ L-methionine and $0.5 \mathrm{ml}$ of $7.8 \mu \mathrm{M}$ riboflavin in order to prepare overall reaction mixture. The reaction was conceded out in similar test tubes at $25^{\circ} \mathrm{C}$ for 15 minutes in 100 $\mu \mathrm{mol}$ photon per $\mathrm{m}^{2}$ fluorescent lamp. Finally, absorbance was recorded with the help of UVVis spectrophotometer at wavelength $645 \mathrm{~nm}$.

Ascorbate peroxidase (APOX) activity was determined as proposed by Janda et al., (1999). $100 \mathrm{mg}$ of plant leaf tissue was crushed in $1 \mathrm{ml}$ of $100 \mathrm{mM}$ HEPES/NaOH buffer (Mol wt.238.3) of pH7.6 containing $8.8 \mathrm{mg}$ acid Ascorbate (Mol. Wt. 176.13) with the help of cooled pestle and mortar. Centrifuged at 10000rpm for 5 minutes and the consequential remaining supernatant were taken as crude extract. Then, took $50 \mu 1$ ascorbate and added $1 \mathrm{ml}$ of $50 \mathrm{mM}$ HEPES buffer followed by $50 \mu 1$ plant extract and finally by mixing it with $100 \mu \mathrm{l}(3 \mathrm{mM})$ hydrogen peroxide and the final reaction mixture was obtained. $50 \mathrm{mM}$ HEPES buffer was used as blank. Absorbance change was recorded at $290 \mathrm{~nm}$ by using UV-Vis spectrophotometer.

Guaiacol peroxidase (GPOX) was assessed by method of Putter (1974). For estimating the GPOX activity, took $3 \mathrm{ml}$ of phosphate buffer then, mixed it with $50 \mathrm{ml}$ of guaiacol solution followed by taking $30 \mathrm{ml}$ of $\mathrm{H}_{2} \mathrm{O}_{2}$ solution and finally, mixed with enzyme crude extract andabsorbance was read at $436 \mathrm{~nm}$.

Catalase (CAT) activity was determined by Aebi (1983) method. In a cuvette, took $1.5 \mathrm{ml}$ of phosphate buffer solution then added $1.2 \mathrm{ml}$ of hydrogen peroxide to it and it was followed by addition of $300 \mu \mathrm{l}$ of enzyme extractand the pace of disintegration of $\mathrm{H} 2 \mathrm{O} 2$ was anticipated spectrophotometrically at $240 \mathrm{~nm}$. From the decrease in absorbance, overall catalase enzyme activity was calculated.

\section{Yield and its attributes}

At the time of harvest, the following yield characteristics were analyzed.

- Number of rows in an ear (NRE) 
- Number of ears per plant (NEP)

- Ear length (EL)

- Biomass

- Grain yield (GY)

\section{RESULTS AND DISCUSSION}

\section{Water relations}

Water potential of leaves $\left(\Psi^{\mathrm{w}}\right)$ showed drastic decrement underneath drought conditions. Water potential of leaves under water stress lowered from -0.62 to $-0.87 \mathrm{Mpa}$ in SUGAR-75 and -0.66 to $-0.83 \mathrm{Mpa}$ in NSC901B at vegetative stage (Table 01). The highest values of water potential (less -ve) were observed at vegetative stage and the least at post silking stage (more -ve). In potassium treated plants, water potential of leaf increased in stressed and non stressed plants. Potassium application increased the water potential in both NSC901B and SUGAR75 genotypes. SUGAR-75 showed higher values of water potential of leaf at all stages (Table 01).

Osmotic potential $\left(\Psi^{\mathrm{s}}\right)$ of leaves decreased (more -ve) with successive growth stages. A considerable decrement in osmotic potential of sweet corn plants was observed at all sampling stages under water deficit stress (Table 02). Potassium treated plants, maintained higher osmotic potential (less -ve) in comparison to control plants in both genotypes. Genotype SUGAR-75 had higher osmotic potential (less -ve) as compared to other genotype (Table 02).

Table 01: $\quad$ Effect of water stress and potassiumon leaf water potential (-MPa) of sweet corn at different growth stages

\begin{tabular}{|c|c|c|c|c|c|c|c|c|c|}
\hline \multirow{3}{*}{ Treatment } & \multicolumn{9}{|c|}{ Sampling stages } \\
\hline & \multicolumn{3}{|c|}{ Vegetative } & \multicolumn{3}{|c|}{ Silking } & \multicolumn{3}{|c|}{ Post silking } \\
\hline & SUGAR-75 & NSC901B & Mean & SUGAR-75 & NSC901B & Mean & SUGAR-75 & NSC901B & Mean \\
\hline FI & $0.621 \pm 0.003$ & $0.661 \pm 0.005$ & 0.641 & $0.691 \pm 0.004$ & $0.721 \pm 0.006$ & 0.706 & $0.751 \pm 0.003$ & $0.774 \pm 0.005$ & 0.762 \\
\hline $\mathrm{FI}+\mathrm{K}_{1}$ & $0.570 \pm 0.009$ & $0.633 \pm 0.012$ & 0.601 & $0.621 \pm 0.006$ & $0.676 \pm 0.009$ & 0.648 & $0.645 \pm 0.005$ & $0.708 \pm 0.008$ & 0.676 \\
\hline $\mathrm{FI}+\mathrm{K}_{2}$ & $0.558 \pm 0.004$ & $0.613 \pm 0.006$ & 0.585 & $0.600 \pm 0.008$ & $0.662 \pm 0.010$ & 0.631 & $0.630 \pm 0.006$ & $0.662 \pm 0.007$ & 0.646 \\
\hline FD & $0.876 \pm 0.007$ & $0.830 \pm 0.009$ & 0.853 & $0.912 \pm 0.007$ & $0.862 \pm 0.009$ & 0.887 & $0.981 \pm 0.009$ & $1.032 \pm 0.011$ & 1.006 \\
\hline $\mathrm{FD}+\mathrm{K}_{1}$ & $0.652 \pm 0.006$ & $0.664 \pm 0.004$ & 0.658 & $0.709 \pm 0.009$ & $0.653 \pm 0.012$ & 0.681 & $0.715 \pm 0.010$ & $0.793 \pm 0.012$ & 0.754 \\
\hline $\mathrm{FD}+\mathrm{K}_{2}$ & $0.591 \pm 0.008$ & $0.614 \pm 0.009$ & 0.602 & $0.664 \pm 0.010$ & $0.610 \pm 0.011$ & 0.637 & $0.637 \pm 0.007$ & $0.721 \pm 0.009$ & 0.679 \\
\hline MED & $0.698 \pm 0.003$ & $0.731 \pm 0.005$ & 0.714 & $0.743 \pm 0.009$ & $0.712 \pm 0.010$ & 0.727 & $0.823 \pm 0.003$ & $0.862 \pm 0.005$ & 0.842 \\
\hline $\mathrm{MED}+\mathrm{K}_{1}$ & $0.586 \pm 0.011$ & $0.591 \pm 0.013$ & 0.588 & $0.599 \pm 0.012$ & $0.539 \pm 0.015$ & 0.569 & $0.639 \pm 0.012$ & $0.705 \pm 0.014$ & 0.672 \\
\hline $\mathrm{MED}+\mathrm{K}_{2}$ & $0.545 \pm 0.004$ & $0.562 \pm 0.006$ & 0.553 & $0.562 \pm 0.006$ & $0.511 \pm 0.007$ & 0.536 & $0.590 \pm 0.004$ & $0.662 \pm 0.006$ & 0.626 \\
\hline SED & $0.798 \pm 0.009$ & $0.834 \pm 0.011$ & 0.816 & $0.851 \pm 0.004$ & $0.910 \pm 0.006$ & 0.880 & $0.931 \pm 0.009$ & $0.096 \pm 0.011$ & 0.513 \\
\hline $\mathrm{SED}+\mathrm{K}_{1}$ & $0.632 \pm 0.007$ & $0.680 \pm 0.009$ & 0.656 & $0.663 \pm 0.011$ & $0.755 \pm 0.013$ & 0.709 & $0.706 \pm 0.009$ & $0.076 \pm 0.013$ & 0.391 \\
\hline $\mathrm{SED}+\mathrm{K}_{2}$ & $0.576 \pm 0.011$ & $0.655 \pm 0.013$ & 0.615 & $0.629 \pm 0.007$ & $0.700 \pm 0.009$ & 0.664 & $0.660 \pm 0.008$ & $0.072 \pm 0.009$ & 0.366 \\
\hline MLD & $0.567 \pm 0.005$ & $0.513 \pm 0.007$ & 0.543 & $0.620 \pm 0.009$ & $0.672 \pm 0.012$ & 0.646 & $0.692 \pm 0.012$ & $0.742 \pm 0.014$ & 0.717 \\
\hline $\mathrm{MLD}+\mathrm{K}_{1}$ & $0.504 \pm 0.007$ & $0.469 \pm 0.009$ & 0.486 & $0.471 \pm 0.005$ & $0.536 \pm 0.007$ & 0.503 & $0.503 \pm 0.004$ & $0.569 \pm 0.006$ & 0.536 \\
\hline $\mathrm{MLD}+\mathrm{K}_{2}$ & $0.492 \pm 0.005$ & $0.459 \pm 0.007$ & 0.475 & $0.458 \pm 0.013$ & $0.515 \pm 0.015$ & 0.486 & $0.483 \pm 0.011$ & $0.547 \pm 0.013$ & 0.515 \\
\hline SLD & $0.653 \pm 0.012$ & $0.702 \pm 0.014$ & 0.677 & $0.691 \pm 0.010$ & $0.765 \pm 0.014$ & 0.728 & $0.781 \pm 0.009$ & $0.843 \pm 0.011$ & 0.812 \\
\hline $\mathrm{SLD}+\mathrm{K}_{1}$ & $0.565 \pm 0.010$ & $0.801 \pm 0.012$ & 0.683 & $0.552 \pm 0.005$ & $0.623 \pm 0.007$ & 0.587 & $0.600 \pm 0.005$ & $0.688 \pm 0.007$ & 0.644 \\
\hline $\mathrm{SLD}+\mathrm{K}_{2}$ & $0.552 \pm 0.014$ & $0.756 \pm 0.016$ & 0.654 & $0.524 \pm 0.008$ & $0.600 \pm 0.010$ & 0.562 & $0.569 \pm 0.007$ & $0.646 \pm 0.009$ & 0.607 \\
\hline Mean & 0.613 & 0.653 & & 0.642 & 0.667 & & 0.685 & 0.622 & \\
\hline \multirow{7}{*}{$\mathrm{CD}$ at $5 \%$} & \multicolumn{3}{|c|}{ Genotype $(\mathrm{G})=0.007$} & \multicolumn{3}{|c|}{ Genotype $(\mathrm{G})=0.007$} & \multicolumn{3}{|c|}{ Genotype $(\mathrm{G})=0.007$} \\
\hline & \multicolumn{3}{|c|}{ Water treatment $(\mathrm{WT})=0.012$} & \multicolumn{3}{|c|}{ Water treatment $(\mathrm{WT})=0.013$} & \multicolumn{3}{|c|}{ Water treatment $(\mathrm{WT})=0.012$} \\
\hline & \multicolumn{3}{|c|}{$\mathrm{G} \times \mathrm{WT}=0.017$} & \multicolumn{3}{|c|}{$\mathrm{G} \times \mathrm{WT}=0.018$} & \multicolumn{3}{|c|}{$\mathrm{G} \times \mathrm{WT}=0.016$} \\
\hline & \multicolumn{3}{|c|}{$\operatorname{Potassium}(\mathrm{K})=0.007$} & \multicolumn{3}{|c|}{$\operatorname{Potassium}(K)=0.007$} & \multicolumn{3}{|c|}{$\operatorname{Potassium}(\mathrm{K})=0.001$} \\
\hline & \multicolumn{3}{|c|}{$\mathrm{G} \times \mathrm{K}=\mathrm{N} / \mathrm{A}$} & \multicolumn{3}{|c|}{$\mathrm{G} \times \mathrm{K}=0.010$} & \multicolumn{3}{|c|}{$\mathrm{G} \times \mathrm{K}=\mathrm{N} / \mathrm{A}$} \\
\hline & \multicolumn{3}{|c|}{$\mathrm{WT} \times \mathrm{K}=0.017$} & \multicolumn{3}{|c|}{$\mathrm{WT} \times \mathrm{K}=0.018$} & \multicolumn{3}{|c|}{$\mathrm{WT} \times \mathrm{K}=0.016$} \\
\hline & \multicolumn{3}{|c|}{$\mathrm{G} \times \mathrm{WT} \times \mathrm{K}=0.024$} & \multicolumn{3}{|c|}{$\mathrm{G} \times \mathrm{WT} \times \mathrm{K}=0.026$} & \multicolumn{3}{|c|}{$\mathrm{G} \times \mathrm{WT} \times \mathrm{K}=0.023$} \\
\hline
\end{tabular}

$F I=$ Fully irrigation, $F D=$ Fully Drought, $M E D=$ Moderate early drought, SED = Severe early drought, $M L D=$ Moderate late drought , $S L D=$ Severe late drought, $K_{1}=500 \mathrm{ppm}$ (potassium treatment), $K_{2}=700 \mathrm{ppm}$ (potassium treatment) 
S. Rao, S. Singh and N. Singh

Table 02: $\quad$ Effect of water stress and potassiumon leaf osmotic potential (-MPa) of sweet corn at different growth stages

\begin{tabular}{|c|c|c|c|c|c|c|c|c|c|}
\hline \multirow{3}{*}{ Treatment } & \multicolumn{9}{|c|}{ Sampling stages } \\
\hline & \multicolumn{3}{|c|}{ Vegetative } & \multicolumn{3}{|c|}{ Silking } & \multicolumn{3}{|c|}{ Post silking } \\
\hline & SUGAR-75 & NSC901B & Mean & SUGAR-75 & NSC901B & Mean & SUGAR-75 & NSC901B & Mean \\
\hline FI & $0.752 \pm 0.005$ & $0.812 \pm 0.007$ & 0.782 & $0.883 \pm 0.009$ & $0.932 \pm 0.012$ & 0.907 & $1.061 \pm 0.006$ & $1.160 \pm 0.008$ & 1.110 \\
\hline $\mathrm{FI}+\mathrm{K}_{1}$ & $0.697 \pm 0.008$ & $0.769 \pm 0.009$ & 0.733 & $0.802 \pm 0.011$ & $0.874 \pm 0.013$ & 0.838 & $0.954 \pm 0.011$ & $1.055 \pm 0.013$ & 1.004 \\
\hline $\mathrm{FI}+\mathrm{K}_{2}$ & $0.661 \pm 0.011$ & $0.745 \pm 0.004$ & 0.703 & $0.783 \pm 0.012$ & $0.846 \pm 0.009$ & 0.814 & $0.922 \pm 0.013$ & $1.032 \pm 0.016$ & 0.977 \\
\hline FD & $0.980 \pm 0.003$ & $1.071 \pm 0.002$ & 1.025 & $1.082 \pm 0.007$ & $1.191 \pm 0.014$ & 1.136 & $1.261 \pm 0.015$ & $1.370 \pm 0.017$ & 1.315 \\
\hline $\mathrm{FD}+\mathrm{K}_{1}$ & $0.744 \pm 0.009$ & $0.856 \pm 0.008$ & 0.801 & $0.777 \pm 0.013$ & $0.904 \pm 0.016$ & 0.840 & $0.945 \pm 0.017$ & $1.082 \pm 0.020$ & 1.013 \\
\hline $\mathrm{FD}+\mathrm{K}_{2}$ & $0.695 \pm 0.004$ & $0.791 \pm 0.006$ & 0.743 & $0.723 \pm 0.008$ & $0.833 \pm 0.010$ & 0.778 & $0.869 \pm 0.009$ & $1.027 \pm 0.013$ & 0.948 \\
\hline MED & $0.842 \pm 0.013$ & $0.973 \pm 0.015$ & 0.907 & $0.961 \pm 0.004$ & $1.072 \pm 0.007$ & 1.016 & $1.131 \pm 0.014$ & $1.280 \pm 0.016$ & 1.205 \\
\hline $\mathrm{MED}+\mathrm{K}_{1}$ & $0.705 \pm 0.010$ & $0.862 \pm 0.011$ & 0.783 & $0.825 \pm 0.014$ & $0.973 \pm 0.016$ & 0.899 & $0.926 \pm 0.011$ & $1.126 \pm 0.013$ & 1.026 \\
\hline $\mathrm{MED}+\mathrm{K}_{2}$ & $0.655 \pm 0.009$ & $0.805 \pm 0.010$ & 0.734 & $0.768 \pm 0.015$ & $0.920 \pm 0.017$ & 0.844 & $0.832 \pm 0.015$ & $1.049 \pm 0.017$ & 0.940 \\
\hline SED & $0.931 \pm 0.011$ & $0.991 \pm 0.013$ & 0.961 & $1.091 \pm 0.017$ & $1.181 \pm 0.019$ & 1.136 & $1.234 \pm 0.009$ & $1.325 \pm 0.011$ & 1.279 \\
\hline $\mathrm{SED}+\mathrm{K}_{1}$ & $0.716 \pm 0.006$ & $0.792 \pm 0.008$ & 0.754 & $0.872 \pm 0.016$ & $0.908 \pm 0.018$ & 0.891 & $0.922 \pm 0.016$ & $1.069 \pm 0.018$ & 0.995 \\
\hline $\mathrm{SED}+\mathrm{K}_{2}$ & $0.678 \pm 0.012$ & $0.752 \pm 0.014$ & 0.715 & $0.828 \pm 0.018$ & $0.861 \pm 0.020$ & 0.844 & $0.861 \pm 0.018$ & $0.976 \pm 0.020$ & 0.918 \\
\hline MLD & $0.783 \pm 0.007$ & $0.843 \pm 0.009$ & 0.813 & $0.890 \pm 0.017$ & $0.952 \pm 0.019$ & 0.921 & $0.972 \pm 0.012$ & $1.121 \pm 0.014$ & 1.046 \\
\hline $\mathrm{MLD}+\mathrm{K}_{1}$ & $0.717 \pm 0.010$ & $0.789 \pm 0.012$ & 0.753 & $0.756 \pm 0.015$ & $0.826 \pm 0.017$ & 0.791 & $0.776 \pm 0.016$ & $0.929 \pm 0.017$ & 0.852 \\
\hline $\mathrm{MLD}+\mathrm{K}_{2}$ & $0.694 \pm 0.009$ & $0.772 \pm 0.013$ & 0.733 & $0.612 \pm 0.012$ & $0.798 \pm 0.014$ & 0.705 & $0.717 \pm 0.019$ & $0.873 \pm 0.021$ & 0.795 \\
\hline SLD & $0.702 \pm 0.016$ & $0.794 \pm 0.018$ & 0.748 & $0.784 \pm 0.019$ & $0.811 \pm 0.021$ & 0.797 & $0.902 \pm 0.021$ & $0.972 \pm 0.025$ & 0.937 \\
\hline $\mathrm{SLD}+\mathrm{K}_{1}$ & $0.623 \pm 0.004$ & $0.726 \pm 0.006$ & 0.674 & $0.624 \pm 0.008$ & $0.672 \pm 0.010$ & 0.648 & $0.701 \pm 0.015$ & $0.814 \pm 0.018$ & 0.757 \\
\hline $\mathrm{SLD}+\mathrm{K}_{2}$ & $0.609 \pm 0.006$ & $0.711 \pm 0.008$ & 0.661 & $0.585 \pm 0.010$ & $0.623 \pm 0.012$ & 0.604 & $0.648 \pm 0.017$ & $0.737 \pm 0.021$ & 0.692 \\
\hline Mean & 0.732 & 0.825 & & 0.813 & 0.898 & & 0.924 & 1.055 & \\
\hline \multirow{7}{*}{$\mathrm{CD}$ at $5 \%$} & \multicolumn{3}{|c|}{$\operatorname{Genotype}(\mathrm{G})=0.009$} & \multicolumn{3}{|c|}{$\operatorname{Genotype}(\mathrm{G})=0.011$} & \multicolumn{3}{|c|}{$\operatorname{Genotype}(\mathrm{G})=0.013$} \\
\hline & \multicolumn{3}{|c|}{ Water treatment $(\mathrm{WT})=0.016$} & \multicolumn{3}{|c|}{ Water treatment $(\mathrm{WT})=0.019$} & \multicolumn{3}{|c|}{ Water treatment $(\mathrm{WT})=0.022$} \\
\hline & \multicolumn{3}{|c|}{$\mathrm{G} \times \mathrm{WT}=0.022$} & \multicolumn{3}{|c|}{$\mathrm{G} \times \mathrm{WT}=0.027$} & \multicolumn{3}{|c|}{$\mathrm{G} \times \mathrm{WT}=0.031$} \\
\hline & \multicolumn{3}{|c|}{$\operatorname{Potassium}(\mathrm{K})=0.009$} & \multicolumn{3}{|c|}{$\operatorname{Potassium}(\mathrm{K})=0.011$} & \multicolumn{3}{|c|}{$\operatorname{Potassium}(\mathrm{K})=0.013$} \\
\hline & \multicolumn{3}{|c|}{$\mathrm{G} \times \mathrm{K}=0.013$} & \multicolumn{3}{|c|}{$\mathrm{G} \times \mathrm{K}=\mathrm{N} / \mathrm{A}$} & \multicolumn{3}{|c|}{$\mathrm{G} \times \mathrm{K}=0.018$} \\
\hline & \multicolumn{3}{|c|}{$\mathrm{WT} \times \mathrm{K}=0.022$} & \multicolumn{3}{|c|}{$\mathrm{WT} \times \mathrm{K}=0.027$} & \multicolumn{3}{|c|}{$\mathrm{WT} \times \mathrm{K}=0.031$} \\
\hline & \multicolumn{3}{|c|}{$\mathrm{G} \times \mathrm{WT} \times \mathrm{K}=0.031$} & \multicolumn{3}{|c|}{$\mathrm{G} \times \mathrm{WT} \times \mathrm{K}=0.038$} & \multicolumn{3}{|c|}{$\mathrm{G} \times \mathrm{WT} \times \mathrm{K}=0.043$} \\
\hline
\end{tabular}

$F I=$ Fully irrigation, $F D=$ Fully Drought, $M E D=$ Moderate early drought, $S E D=$ Severe early drought, $M L D=$ Moderate late drought, $S L D=$ Severe late drought, $K_{1}=500 \mathrm{ppm}$ ( potassium treatment), $K_{2}=700 \mathrm{ppm}$ (potassium treatment)

Relative water content (RWC) of foliage showed decrement from vegetative to post silking stage in both the genotypes (Table 03). A significant enhancement in RWC of foliage was observed by increasing concentration of potassium under normal and stressed conditions. SUGAR-75 maintained higher relative water content as evaluate to other cultivar irrespective of growth stage (Table 03).

Alteration in osmotic solutes is a function of cultivars, growth stage and intensity and severity of drought (Zhang et al., 2013). In this investigation decrease in water potential, osmotic potential and relative water content was noted in water deficitconditions. This may be due 
to decrease in availability of water, absorption and translocation of water from soil to roots and finally, to shoots (Clavel et al., 2005). Increase in free amino acids and decrease in protein degradation under water deficit due to enhanced proteolase activity results in reduction in RWC (Zhang et al., 2009).

Potassium acts as a crucial osmoprotectant and maintains the turgor pressure of leaf results in overall increase in yield (Cakmak, 2005). Most of the plants accumulate active osmolytes in drought stress referred as osmotic adjustment (Afkari et al., 2009). Increase in RWC by application of potassium fertilizer was reported in maize (Zhang et al., 2014) and it indicates that accumulation of potassium maintains the water balance, osmotic adjustment, turgor pressure and many other physiological processes of plants, subjected to drought stress (Serraj and Sinclair, 2002). Application of potassium in water deficit conditions helps in osmotic adjustment of plants (Jain et al., 2019). Raza et al., (2014) recorded that osmotic potential was found to be less negative in plants treated with potassium, compared to non-treated plants under low moisture stress.

Table 03: $\quad$ Effect of water stress and potassium on relative water content (\%) of sweet corn at different growth stages

\begin{tabular}{|c|c|c|c|c|c|c|c|c|c|}
\hline \multirow{3}{*}{ Treatment } & \multicolumn{9}{|c|}{ Sampling stages } \\
\hline & \multicolumn{3}{|c|}{ Vegetative } & \multicolumn{3}{|c|}{ Silking } & \multicolumn{3}{|c|}{ Post silking } \\
\hline & SUGAR-75 & NSC901B & Mean & SUGAR-75 & NSC901B & Mean & SUGAR-75 & NSC901B & Mean \\
\hline FI & $55.21 \pm 0.326$ & $53.28 \pm 0.912$ & 54.24 & $51.98 \pm 0.269$ & $49.94 \pm 0.115$ & 50.96 & $45.82 \pm 0.179$ & $43.19 \pm 0.141$ & 44.50 \\
\hline $\mathrm{FI}+\mathrm{K}_{1}$ & $60.73 \pm 0.564$ & $57.08 \pm 0.437$ & 58.90 & $57.69 \pm 0.466$ & $54.43 \pm 0.529$ & 56.06 & $47.65 \pm 0.302$ & $44.48 \pm 0.279$ & 46.06 \\
\hline $\mathrm{FI}+\mathrm{K}_{2}$ & $64.04 \pm 0.798$ & $60.20 \pm 0.132$ & 62.12 & $60.28 \pm 0.659$ & $56.63 \pm 0.432$ & 58.45 & $48.56 \pm 0.423$ & $45.34 \pm 0.321$ & 46.95 \\
\hline FD & $35.39 \pm 0.317$ & $32.50 \pm 0.119$ & 33.94 & $22.86 \pm 0.266$ & $20.45 \pm 0.116$ & 21.65 & $17.41 \pm 0.164$ & $14.68 \pm 0.126$ & 16.04 \\
\hline $\mathrm{FD}+\mathrm{K}_{1}$ & $38.57 \pm 0.461$ & $33.83 \pm 0.127$ & 36.29 & $25.83 \pm 0.380$ & $20.69 \pm 0.289$ & 23.26 & $17.58 \pm 0.253$ & $14.82 \pm 0.204$ & 16.24 \\
\hline $\mathrm{FD}+\mathrm{K}_{2}$ & $37.86 \pm 0.713$ & $34.45 \pm 0.329$ & 36.15 & $23.77 \pm 0.658$ & $20.83 \pm 0.432$ & 22.31 & $17.75 \pm 0.424$ & $14.89 \pm 0.317$ & 16.32 \\
\hline MED & $35.88 \pm 1.128$ & $31.96 \pm 0.218$ & 33.92 & $38.46 \pm 0.932$ & $34.95 \pm 0.654$ & 36.70 & $22.91 \pm 0.319$ & $19.86 \pm 0.263$ & 21.38 \\
\hline $\mathrm{MED}+\mathrm{K}_{1}$ & $42.69 \pm 0.230$ & $37.07 \pm 0.116$ & 39.88 & $48.07 \pm 0.190$ & $43.33 \pm 0.110$ & 45.79 & $26.34 \pm 0.123$ & $22.04 \pm 0.117$ & 24.19 \\
\hline $\mathrm{MED}+\mathrm{K}_{2}$ & $45.92 \pm 0.399$ & $39.63 \pm 0.145$ & 42.77 & $51.92 \pm 0.329$ & $45.78 \pm 0.216$ & 48.85 & $27.72 \pm 0.218$ & $23.23 \pm 0.201$ & 25.47 \\
\hline SED & $30.91 \pm 0.564$ & $25.04 \pm 0.212$ & 27.97 & $35.86 \pm 0.466$ & $32.46 \pm 0.312$ & 34.16 & $20.16 \pm 0.313$ & $17.27 \pm 0.295$ & 18.71 \\
\hline $\mathrm{SED}+\mathrm{K}_{1}$ & $34.63 \pm 0.230$ & $27.54 \pm 0.119$ & 31.08 & $41.95 \pm 0.190$ & $41.87 \pm 0.117$ & 41.91 & $21.97 \pm 0.128$ & $18.30 \pm 0.114$ & 20.13 \\
\hline $\mathrm{SED}+\mathrm{K}_{2}$ & $35.54 \pm 0.326$ & $28.29 \pm 0.154$ & 31.91 & $43.74 \pm 0.269$ & $41.54 \pm 0.127$ & 42.64 & $22.37 \pm 0.173$ & $18.47 \pm 0.162$ & 20.42 \\
\hline MLD & $54.32 \pm 0.564$ & $52.87 \pm 0.237$ & 53.59 & $47.82 \pm 0.115$ & $43.94 \pm 0.110$ & 45.88 & $38.48 \pm 0.311$ & $34.98 \pm 0.221$ & 36.73 \\
\hline $\mathrm{MLD}+\mathrm{K}_{1}$ & $58.66 \pm 0.230$ & $57.62 \pm 0.128$ & 58.14 & $58.81 \pm 0.659$ & $52.72 \pm 0.321$ & 55.76 & $42.71 \pm 0.426$ & $39.52 \pm 0.362$ & 40.53 \\
\hline $\mathrm{MLD}+\mathrm{K}_{2}$ & $62.47 \pm 0.326$ & $59.74 \pm 0.290$ & 61.10 & $61.68 \pm 0.213$ & $55.36 \pm 0.203$ & 58.52 & $44.25 \pm 0.232$ & $38.82 \pm 0.210$ & 41.53 \\
\hline SLD & $55.89 \pm 0.564$ & $52.39 \pm 0.251$ & 54.14 & $41.58 \pm 0.116$ & $36.45 \pm 0.102$ & 39.01 & $30.69 \pm 0.119$ & $27.20 \pm 0.110$ & 28.94 \\
\hline $\mathrm{SLD}+\mathrm{K}_{1}$ & $60.92 \pm 0.329$ & $55.97 \pm 0.312$ & 58.43 & $49.48 \pm 0.212$ & $42.28 \pm 0.126$ & 42.96 & $33.75 \pm 0.171$ & $29.37 \pm 0.162$ & 31.56 \\
\hline $\mathrm{SLD}+\mathrm{K}_{2}$ & $62.59 \pm 0.113$ & $58.06 \pm 0.110$ & 60.32 & $51.97 \pm 0.342$ & $44.46 \pm 0.219$ & 48.21 & $32.83 \pm 0.413$ & $28.28 \pm 0.321$ & 30.55 \\
\hline Mean & 48.45 & 44.30 & & 45.20 & 41.06 & & 31.05 & 25.85 & \\
\hline \multirow{7}{*}{$\mathrm{CD}$ at $5 \%$} & \multicolumn{3}{|c|}{ Genotype $(\mathrm{G})=0.655$} & \multicolumn{3}{|c|}{ Genotype $(\mathrm{G})=0.541$} & \multicolumn{3}{|c|}{ Genotype $(\mathrm{G})=0.359$} \\
\hline & \multicolumn{3}{|c|}{ Water treatment $(\mathrm{WT})=1.135$} & \multicolumn{3}{|c|}{ Water treatment $(\mathrm{WT})=0.937$} & \multicolumn{3}{|c|}{ Water treatment $(\mathrm{WT})=0.622$} \\
\hline & \multicolumn{3}{|c|}{$\mathrm{G} \times \mathrm{WT}=1.605$} & \multicolumn{3}{|c|}{$\mathrm{G} \times \mathrm{WT}=1.325$} & \multicolumn{3}{|c|}{$\mathrm{G} \times \mathrm{WT}=0.880$} \\
\hline & \multicolumn{3}{|c|}{$\operatorname{Potassium}(\mathrm{K})=0.655$} & \multicolumn{3}{|c|}{$\operatorname{Potassium}(K)=0.541$} & \multicolumn{3}{|c|}{$\operatorname{Potassium}(K)=0.359$} \\
\hline & \multicolumn{3}{|c|}{$\mathrm{G} \times \mathrm{K}=\mathrm{N} / \mathrm{A}$} & \multicolumn{3}{|c|}{$\mathrm{G} \times \mathrm{K}=\mathrm{N} / \mathrm{A}$} & \multicolumn{3}{|c|}{$\mathrm{G} \times \mathrm{K}=0.508$} \\
\hline & \multicolumn{3}{|c|}{$\mathrm{WT} \times \mathrm{K}=1.605$} & \multicolumn{3}{|c|}{$\mathrm{WT} \times \mathrm{K}=1.325$} & \multicolumn{3}{|c|}{$\mathrm{WT} \times \mathrm{K}=0.880$} \\
\hline & \multicolumn{3}{|c|}{$\mathrm{G} \times \mathrm{WT} \times \mathrm{K}=2.269$} & \multicolumn{3}{|c|}{$\mathrm{G} \times \mathrm{WT} \times \mathrm{K}=1.874$} & \multicolumn{3}{|c|}{$\mathrm{G} \times \mathrm{WT} \times \mathrm{K}=1.244$} \\
\hline
\end{tabular}

$F I=$ Fully irrigation, $F D=$ Fully Drought, $M E D=$ Moderate early drought, $S E D=$ Severe early drought, $M L D=$ Moderate late drought , $S L D=$ Severe late drought, $K_{1}=500 \mathrm{ppm}$ ( potassium treatment), $K_{2}=700 \mathrm{ppm}$ (potassium treatment) 


\section{Antioxidant enzymes activities}

Superoxide dismutase (SOD) activity increased in all stages with the increasing drought stress. The SOD activity of leaves was highest at vegetative stage then, declined at subsequent stages. SOD activity was found to be relatively more in SUGAR-75 in contrast to NSC901B in non-stressed as well as stressed conditions (Table 04).

A noteworthy increment in SOD of leaves was observed with potassium application in both the cultivars irrespective of sampling stage. Maximum increase of $66 \%$ was observed in genotype SUGAR-75 over test control of fully drought at silking stage plants treated with $700 \mathrm{ppm}$ potassium concentration.

The GPOX activity of leaves decreased from vegetative to silking and then, sharply increased at post silking stage. Water stress resulted in higher GPOX activity in both genotypes at all sampling stages. GPOX activity was relatively greater in SUGAR-75 in contrast to NSC901B under control as well as stress condition. Highest increment of $76 \%$ was observed over test control of fully drought at silking stage under $700 \mathrm{ppm}$ concentration of potassium (Table 05).

Table 04: $\quad$ Effect of water stress and potassium on the activity of SOD (UAmg ${ }^{-1}$ protein) in sweet corn at different growth stages

\begin{tabular}{|c|c|c|c|c|c|c|c|c|c|}
\hline \multirow{3}{*}{ Treatment } & \multicolumn{9}{|c|}{ Sampling stages } \\
\hline & \multicolumn{3}{|c|}{ Vegetative } & \multicolumn{3}{|c|}{ Silking } & \multicolumn{3}{|c|}{ Post silking } \\
\hline & SUGAR-75 & NSC901B & Mean & SUGAR-75 & NSC901B & Mean & SUGAR-75 & NSC901B & Mean \\
\hline FI & $6.889 \pm 0.079$ & $5.623 \pm 0.056$ & 6.256 & $4.278 \pm 0.067$ & $3.924 \pm 0.034$ & 4.101 & $2.268 \pm 0.022$ & $2.076 \pm 0.019$ & 2.172 \\
\hline $\mathrm{FI}+\mathrm{K}_{1}$ & $7.497 \pm 0.138$ & $6.463 \pm 0.124$ & 6.980 & $5.337 \pm 0.116$ & $4.464 \pm 0.104$ & 4.905 & $2.531 \pm 0.031$ & $2.442 \pm 0.028$ & 2.486 \\
\hline $\mathrm{FI}+\mathrm{K}_{2}$ & $7.843 \pm 0.195$ & $6.856 \pm 0.163$ & 7.349 & $5.593 \pm 0.164$ & $4.761 \pm 0.129$ & 5.177 & $2.599 \pm 0.052$ & $2.504 \pm 0.049$ & 2.551 \\
\hline FD & $13.20 \pm 0.079$ & $9.947 \pm 0.034$ & 11.57 & $7.728 \pm 0.038$ & $7.105 \pm 0.020$ & 7.416 & $3.435 \pm 0.036$ & $2.856 \pm 0.031$ & 3.145 \\
\hline $\mathrm{FD}+\mathrm{K}_{1}$ & $19.53 \pm 0.112$ & $14.12 \pm 0.110$ & 16.82 & $11.97 \pm 0.094$ & $10.58 \pm 0.047$ & 11.27 & $4.396 \pm 0.031$ & $3.455 \pm 0.027$ & 3.925 \\
\hline $\mathrm{FD}+\mathrm{K}_{2}$ & $21.25 \pm 0.186$ & $15.51 \pm 0.154$ & 18.38 & $12.82 \pm 0.167$ & $11.43 \pm 0.132$ & 12.12 & $4.637 \pm 0.057$ & $3.741 \pm 0.048$ & 4.189 \\
\hline MED & $15.06 \pm 0.275$ & $10.79 \pm 0.212$ & 12.92 & $8.411 \pm 0.231$ & $6.770 \pm 0.210$ & 7.595 & $3.887 \pm 0.076$ & $3.332 \pm 0.056$ & 3.609 \\
\hline $\mathrm{MED}+\mathrm{K}_{1}$ & $18.82 \pm 0.056$ & $13.05 \pm 0.037$ & 15.93 & $11.18 \pm 0.047$ & $8.327 \pm 0.022$ & 9.753 & $5.014 \pm 0.015$ & $4.098 \pm 0.013$ & 4.556 \\
\hline $\mathrm{MED}+\mathrm{K}_{2}$ & $20.02 \pm 0.097$ & $13.81 \pm 0.047$ & 16.91 & $11.94 \pm 0.080$ & $9.342 \pm 0.054$ & 10.64 & $5.247 \pm 0.027$ & $4.364 \pm 0.024$ & 4.805 \\
\hline SED & $16.30 \pm 0.129$ & $12.30 \pm 0.121$ & 14.30 & $9.137 \pm 0.119$ & $7.254 \pm 0.105$ & 8.195 & $3.638 \pm 0.036$ & $3.063 \pm 0.017$ & 3.350 \\
\hline $\mathrm{SED}+\mathrm{K}_{1}$ & $21.51 \pm 0.036$ & $15.49 \pm 0.029$ & 18.51 & $12.42 \pm 0.042$ & $9.357 \pm 0.026$ & 10.88 & $4.547 \pm 0.017$ & $3.706 \pm 0.013$ & 4.126 \\
\hline $\mathrm{SED}+\mathrm{K}_{2}$ & $23.79 \pm 0.123$ & $17.58 \pm 0.110$ & 20.68 & $13.88 \pm 0.113$ & $10.73 \pm 0.119$ & 12.30 & $4.911 \pm 0.083$ & $4.012 \pm 0.065$ & 4.461 \\
\hline MLD & $6.598 \pm 0.324$ & $5.286 \pm 0.313$ & 5.942 & $6.917 \pm 0.129$ & $5.654 \pm 0.121$ & 6.285 & $3.796 \pm 0.276$ & $3.312 \pm 0.241$ & 3.554 \\
\hline $\mathrm{MLD}+\mathrm{K}_{1}$ & $7.380 \pm 0.212$ & $5.649 \pm 0.229$ & 6.514 & $8.922 \pm 0.118$ & $7.010 \pm 0.113$ & 7.966 & $5.124 \pm 0.091$ & $4.371 \pm 0.087$ & 4.747 \\
\hline $\mathrm{MLD}+\mathrm{K}_{2}$ & $7.117 \pm 0.318$ & $6.019 \pm 0.261$ & 6.568 & $9.545 \pm 0.231$ & $7.519 \pm 0.212$ & 8.532 & $5.428 \pm 0.052$ & $4.603 \pm 0.039$ & 5.015 \\
\hline SLD & $6.713 \pm 0.141$ & $5.713 \pm 0.191$ & 6.213 & $7.472 \pm 0.143$ & $6.212 \pm 0.126$ & 6.842 & $3.525 \pm 0.153$ & $3.042 \pm 0.127$ & 3.283 \\
\hline $\mathrm{SLD}+\mathrm{K}_{1}$ & $7.179 \pm 0.283$ & $6.452 \pm 0.182$ & 6.815 & $10.53 \pm 0.212$ & $8.261 \pm 0.142$ & 9.395 & $4.653 \pm 0.117$ & $3.802 \pm 0.110$ & 4.227 \\
\hline $\mathrm{SLD}+\mathrm{K}_{2}$ & $7.448 \pm 0.312$ & $6.737 \pm 0.279$ & 7.092 & $11.50 \pm 0.142$ & $9.193 \pm 0.101$ & 10.34 & $5.146 \pm 0.213$ & $4.258 \pm 0.210$ & 4.702 \\
\hline Mean & 13.08 & 9.855 & & 9.421 & 7.660 & & 4.154 & 3.502 & \\
\hline \multirow{7}{*}{$\mathrm{CD}$ at $5 \%$} & \multicolumn{3}{|c|}{$\operatorname{Genotype}(G)=0.160$} & \multicolumn{3}{|c|}{ Genotype $(G)=0.134$} & \multicolumn{3}{|c|}{ Genotype $(G)=0.044$} \\
\hline & \multicolumn{3}{|c|}{ Water treatment $(\mathrm{WT})=0.277$} & \multicolumn{3}{|c|}{ Water treatment $(\mathrm{WT})=0.233$} & \multicolumn{3}{|c|}{ Water treatment $(\mathrm{WT})=0.076$} \\
\hline & \multicolumn{3}{|c|}{$\mathrm{G} \times \mathrm{WT}=0.391$} & \multicolumn{3}{|c|}{$\mathrm{G} \times \mathrm{WT}=0.329$} & \multicolumn{3}{|c|}{$\mathrm{G} \times \mathrm{WT}=0.108$} \\
\hline & \multicolumn{3}{|c|}{$\operatorname{Potassium}(\mathrm{K})=0.160$} & \multicolumn{3}{|c|}{$\operatorname{Potassium}(\mathrm{K})=0.134$} & \multicolumn{3}{|c|}{$\operatorname{Potassium}(\mathrm{K})=0.044$} \\
\hline & \multicolumn{3}{|c|}{$\mathrm{G} \times \mathrm{K}=0.226$} & \multicolumn{3}{|c|}{$\mathrm{G} \times \mathrm{K}=\mathrm{N} / \mathrm{A}$} & \multicolumn{3}{|c|}{$\mathrm{G} \times \mathrm{K}=\mathrm{N} / \mathrm{A}$} \\
\hline & \multicolumn{3}{|c|}{$\mathrm{WT} \times \mathrm{K}=0.391$} & \multicolumn{3}{|c|}{$\mathrm{WT} \times \mathrm{K}=0.329$} & \multicolumn{3}{|c|}{$\mathrm{WT} \times \mathrm{K}=0.108$} \\
\hline & \multicolumn{3}{|c|}{$\mathrm{G} \times \mathrm{WT} \times \mathrm{K}=0.554$} & \multicolumn{3}{|c|}{$\mathrm{G} \times \mathrm{WT} \times \mathrm{K}=0.466$} & \multicolumn{3}{|c|}{$\mathrm{G} \times \mathrm{WT} \times \mathrm{K}=0.152$} \\
\hline
\end{tabular}

$F I=$ Fully irrigation, $F D=$ Fully Drought, $M E D=$ Moderate early drought, $S E D=$ Severe early drought, $M L D=$ Moderate late drought , $S L D=$ Severe late drought, $K_{1}=500 \mathrm{ppm}$ (potassium treatment),$K_{2}=700 \mathrm{ppm}$ (potassium treatment) 
Table 05: $\quad$ Effect of water stress and potassium on GPOX activity (UAmg-1 protein) of sweet corn at different growth stages

\begin{tabular}{|c|c|c|c|c|c|c|c|c|c|}
\hline \multirow{3}{*}{ Treatment } & \multicolumn{9}{|c|}{ Sampling stages } \\
\hline & \multicolumn{3}{|c|}{ Vegetative } & \multicolumn{3}{|c|}{ Silking } & \multicolumn{3}{|c|}{ Post silking } \\
\hline & SUGAR-75 & NSC901B & Mean & SUGAR-75 & NSC901B & Mean & SUGAR-75 & NSC901B & Mean \\
\hline FI & $4.197 \pm 0.060$ & $3.712 \pm 0.043$ & 3.954 & $2.826 \pm 0.067$ & $2.487 \pm 0.423$ & 2.656 & $8.569 \pm 0.155$ & $7.883 \pm 0.122$ & 8.226 \\
\hline $\mathrm{FI}+\mathrm{K}_{1}$ & $5.028 \pm 0.103$ & $4.637 \pm 0.101$ & 4.832 & $3.158 \pm 0.115$ & $2.852 \pm 0.107$ & 3.005 & $9.159 \pm 0.268$ & $8.825 \pm 0.209$ & 8.992 \\
\hline $\mathrm{FI}+\mathrm{K}_{2}$ & $5.530 \pm 0.146$ & $4.785 \pm 0.124$ & 5.157 & $3.468 \pm 0.163$ & $2.926 \pm 0.149$ & 3.197 & $10.01 \pm 0.380$ & $9.534 \pm 0.231$ & 9.772 \\
\hline FD & $12.98 \pm 0.060$ & $10.83 \pm 0.047$ & 11.90 & $10.15 \pm 0.059$ & $8.308 \pm 0.039$ & 9.229 & $23.96 \pm 0.149$ & $23.24 \pm 0.120$ & 23.61 \\
\hline $\mathrm{FD}+\mathrm{K}_{1}$ & $19.85 \pm 0.084$ & $15.27 \pm 0.039$ & 17.56 & $16.13 \pm 0.094$ & $12.29 \pm 0.076$ & 14.21 & $32.34 \pm 0.118$ & $30.21 \pm 0.111$ & 31.27 \\
\hline $\mathrm{FD}+\mathrm{K}_{2}$ & $20.89 \pm 0.146$ & $16.89 \pm 0.128$ & 18.89 & $17.86 \pm 0.152$ & $14.12 \pm 0.120$ & 15.99 & $36.89 \pm 0.162$ & $33.93 \pm 0.149$ & 35.41 \\
\hline MED & $9.637 \pm 0.207$ & $8.087 \pm 0.103$ & 8.862 & $7.332 \pm 0.230$ & $6.026 \pm 0.212$ & 6.679 & $21.05 \pm 0.142$ & $20.09 \pm 0.132$ & 20.57 \\
\hline $\mathrm{MED}+\mathrm{K}_{1}$ & $13.20 \pm 0.042$ & $10.90 \pm 0.037$ & 12.05 & $10.48 \pm 0.117$ & $8.014 \pm 0.105$ & 9.247 & $27.57 \pm 0.117$ & $24.71 \pm 0.109$ & 26.14 \\
\hline $\mathrm{MED}+\mathrm{K}_{2}$ & $14.26 \pm 0.073$ & $11.32 \pm 0.082$ & 12.79 & $11.36 \pm 0.048$ & $8.918 \pm 0.032$ & 10.13 & $29.89 \pm 0.231$ & $27.52 \pm 0.211$ & 28.70 \\
\hline SED & $11.31 \pm 0.103$ & $9.386 \pm 0.105$ & 10.34 & $8.742 \pm 0.081$ & $7.415 \pm 0.078$ & 8.078 & $19.68 \pm 0.382$ & $17.25 \pm 0.276$ & 18.46 \\
\hline $\mathrm{SED}+\mathrm{K}_{1}$ & $16.73 \pm 0.040$ & $13.14 \pm 0.028$ & 14.93 & $13.46 \pm 0.112$ & $10.23 \pm 0.110$ & 11.84 & $26.96 \pm 0.256$ & $21.73 \pm 0.231$ & 24.34 \\
\hline $\mathrm{SED}+\mathrm{K}_{2}$ & $17.64 \pm 0.068$ & $14.17 \pm 0.049$ & 15.90 & $14.42 \pm 0.044$ & $11.34 \pm 0.032$ & 12.88 & $28.53 \pm 0.219$ & $24.32 \pm 0.201$ & 26.42 \\
\hline MLD & $4.323 \pm 0.072$ & $3.432 \pm 0.105$ & 3.877 & $6.796 \pm 0.041$ & $6.150 \pm 0.027$ & 6.473 & $19.77 \pm 0.039$ & $17.02 \pm 0.027$ & 18.39 \\
\hline $\mathrm{MLD}+\mathrm{K}_{1}$ & $4.968 \pm 0.059$ & $3.773 \pm 0.209$ & 4.370 & $8.630 \pm 0.149$ & $7.380 \pm 0.103$ & 8.005 & $24.31 \pm 0.405$ & $20.08 \pm 0.209$ & 22.19 \\
\hline $\mathrm{MLD}+\mathrm{K}_{2}$ & $5.420 \pm 0.041$ & $4.150 \pm 0.330$ & 4.785 & $9.650 \pm 0.231$ & $8.487 \pm 0.291$ & 9.068 & $27.87 \pm 0.352$ & $24.67 \pm 0.273$ & 26.27 \\
\hline SLD & $4.063 \pm 0.036$ & $3.895 \pm 0.286$ & 3.979 & $7.952 \pm 0.129$ & $6.745 \pm 0.120$ & 7.348 & $21.57 \pm 0.276$ & $18.83 \pm 0.231$ & 20.27 \\
\hline $\mathrm{SLD}+\mathrm{K}_{1}$ & $4.831 \pm 0.042$ & $4.473 \pm 0.374$ & 4.652 & $11.29 \pm 0.342$ & $9.173 \pm 0.311$ & 10.23 & $29.11 \pm 0.321$ & $26.73 \pm 0.113$ & 27.92 \\
\hline $\mathrm{SLD}+\mathrm{K}_{2}$ & $5.237 \pm 0.032$ & $4.862 \pm 0.021$ & 5.049 & $12.16 \pm 0.274$ & $8.950 \pm 0.265$ & 10.55 & $30.84 \pm 0.276$ & $28.43 \pm 0.251$ & 29.63 \\
\hline Mean & 10.05 & 8.206 & & 9.770 & 7.878 & & 23.78 & 21.38 & \\
\hline \multirow{7}{*}{$\mathrm{CD}$ at $5 \%$} & \multicolumn{3}{|c|}{ Genotype $(G)=0.120$} & \multicolumn{3}{|c|}{ Genotype $(G)=0.134$} & \multicolumn{3}{|c|}{ Genotype $(\mathrm{G})=0.312$} \\
\hline & \multicolumn{3}{|c|}{ Water treatment $(\mathrm{WT})=0.208$} & \multicolumn{3}{|c|}{ Water treatment $(\mathrm{WT})=0.232$} & \multicolumn{3}{|c|}{ Water treatment $(\mathrm{WT})=0.540$} \\
\hline & \multicolumn{3}{|c|}{$\mathrm{G} \times \mathrm{WT}=0.294$} & \multicolumn{3}{|c|}{$\mathrm{G} \times \mathrm{WT}=0.328$} & \multicolumn{3}{|c|}{$\mathrm{G} \times \mathrm{WT}=0.763$} \\
\hline & \multicolumn{3}{|c|}{$\operatorname{Potassium}(\mathrm{K})=0.120$} & \multicolumn{3}{|c|}{$\operatorname{Potassium}(\mathrm{K})=0.134$} & \multicolumn{3}{|c|}{$\operatorname{Potassium}(\mathrm{K})=0.312$} \\
\hline & \multicolumn{3}{|c|}{$\mathrm{G} \times \mathrm{K}=0.170$} & \multicolumn{3}{|c|}{$\mathrm{G} \times \mathrm{K}=0.189$} & \multicolumn{3}{|c|}{$\mathrm{G} \times \mathrm{K}=\mathrm{N} / \mathrm{A}$} \\
\hline & \multicolumn{3}{|c|}{$\mathrm{WT} \times \mathrm{K}=0.294$} & \multicolumn{3}{|c|}{$\mathrm{WT} \times \mathrm{K}=0.328$} & \multicolumn{3}{|c|}{$\mathrm{WT} \times \mathrm{K}=0.763$} \\
\hline & \multicolumn{3}{|c|}{$\mathrm{G} \times \mathrm{WT} \times \mathrm{K}=0.416$} & \multicolumn{3}{|c|}{$\mathrm{G} \times \mathrm{WT} \times \mathrm{K}=0.463$} & \multicolumn{3}{|c|}{$\mathrm{G} \times \mathrm{WT} \times \mathrm{K}=1.079$} \\
\hline
\end{tabular}

$F I=$ Fully irrigation, $F D=$ Fully Drought, $M E D=$ Moderate early drought, $S E D=$ Severe early drought, $M L D=$ Moderate late drought , $S L D=$ Severe late drought, $K_{1}=500 \mathrm{ppm}$ ( potassium treatment), $K_{2}=700 \mathrm{ppm}$ (potassium treatment)

A remarkable increment in activity of APOX was noticed from vegetative to silking stage and then, declined at post silking stage in both cultivars. Maximum increase of 58\% was observed over test control of severe early drought at vegetative stage of genotype SUGAR-75. Application of potassium further increased the APOX activity. The maximum enhancement of $69 \%$ was observed over test control of fully drought at silking stage, under 700ppm concentration of potassium (Table 06). Among genotypes, SUGAR-75 had higher accumulation of enzymatic activity than NSC901B at all sampling stage.
Catalase (CAT) activity progressively decreased in all sampling stages with the advancement of time in both cultivars. Enhanced activity of CAT was observed in both cultivars over water deficit conditions. CAT activity was found to be further enhanced with the application of potassium in both the cultivars (Table 07). However, the best response was observed with 700ppm potassium in genotype SUGAR-75 at severe early drought. 
S. Rao, S. Singh and N. Singh

Table 06: $\quad$ Effect of water stress and potassium on APOX activity (UAmg-1protein) of sweet corn at different growth stages

\begin{tabular}{|c|c|c|c|c|c|c|c|c|c|}
\hline \multirow{3}{*}{ Treatment } & \multicolumn{9}{|c|}{ Sampling stages } \\
\hline & \multicolumn{3}{|c|}{ Vegetative } & \multicolumn{3}{|c|}{ Silking } & \multicolumn{3}{|c|}{ Post silking } \\
\hline & SUGAR-75 & NSC901B & Mean & SUGAR-75 & NSC901B & Mean & SUGAR-75 & NSC901B & Mean \\
\hline FI & $2.875 \pm 0.023$ & $2.513 \pm 0.021$ & 2.694 & $3.287 \pm 0.043$ & $2.973 \pm 0.034$ & 3.130 & $0.925 \pm 0.009$ & $1.213 \pm 0.007$ & 1.069 \\
\hline $\mathrm{FI}+\mathrm{K}_{1}$ & $3.309 \pm 0.040$ & $2.811 \pm 0.028$ & 3.060 & $4.198 \pm 0.075$ & $3.682 \pm 0.068$ & 3.941 & $1.104 \pm 0.015$ & $1.512 \pm 0.012$ & 1.308 \\
\hline $\mathrm{FI}+\mathrm{K}_{2}$ & $3.530 \pm 0.057$ & $3.037 \pm 0.040$ & 3.283 & $4.460 \pm 0.107$ & $3.861 \pm 0.101$ & 4.160 & $1.177 \pm 0.021$ & $1.597 \pm 0.018$ & 1.387 \\
\hline FD & $3.644 \pm 0.043$ & $3.212 \pm 0.041$ & 3.428 & $5.412 \pm 0.051$ & $4.533 \pm 0.043$ & 4.972 & $1.131 \pm 0.030$ & $1.609 \pm 0.027$ & 1.372 \\
\hline $\mathrm{FD}+\mathrm{K}_{1}$ & $5.174 \pm 0.059$ & $4.409 \pm 0.054$ & 4.791 & $8.226 \pm 0.154$ & $6.452 \pm 0.138$ & 7.339 & $1.606 \pm 0.006$ & $2.172 \pm 0.004$ & 1.889 \\
\hline $\mathrm{FD}+\mathrm{K}_{2}$ & $5.939 \pm 0.081$ & $5.074 \pm 0.022$ & 5.506 & $9.143 \pm 0.122$ & $7.311 \pm 0.119$ & 8.227 & $1.767 \pm 0.019$ & $2.349 \pm 0.021$ & 2.058 \\
\hline MED & $3.616 \pm 0.017$ & $3.313 \pm 0.015$ & 3.464 & $4.329 \pm 0.079$ & $3.593 \pm 0.067$ & 3.961 & $1.068 \pm 0.024$ & $1.512 \pm 0.027$ & 1.291 \\
\hline $\mathrm{MED}+\mathrm{K}_{1}$ & $4.647 \pm 0.029$ & $4.141 \pm 0.018$ & 4.394 & $5.930 \pm 0.056$ & $4.742 \pm 0.043$ & 5.336 & $1.407 \pm 0.017$ & $1.874 \pm 0.020$ & 1.640 \\
\hline $\mathrm{MED}+\mathrm{K}_{2}$ & $4.953 \pm 0.051$ & $4.472 \pm 0.043$ & 4.712 & $6.233 \pm 0.039$ & $5.030 \pm 0.037$ & 5.631 & $1.504 \pm 0.025$ & $2.041 \pm 0.028$ & 1.772 \\
\hline SED & $4.534 \pm 0.046$ & $3.714 \pm 0.041$ & 4.124 & $4.952 \pm 0.103$ & $4.217 \pm 0.010$ & 4.584 & $1.186 \pm 0.027$ & $1.464 \pm 0.029$ & 1.325 \\
\hline $\mathrm{SED}+\mathrm{K}_{1}$ & $7.027 \pm 0.062$ & $5.571 \pm 0.054$ & 6.299 & $7.428 \pm 0.029$ & $6.156 \pm 0.021$ & 6.792 & $1.802 \pm 0.018$ & $2.093 \pm 0.020$ & 1.947 \\
\hline $\mathrm{SED}+\mathrm{K}_{2}$ & $6.710 \pm 0.031$ & $5.311 \pm 0.028$ & 6.010 & $7.972 \pm 0.016$ & $6.578 \pm 0.011$ & 7.275 & $1.838 \pm 0.025$ & $2.196 \pm 0.027$ & 2.017 \\
\hline MLD & $2.984 \pm 0.064$ & $2.335 \pm 0.061$ & 2.659 & $4.165 \pm 0.045$ & $4.009 \pm 0.042$ & 4.087 & $1.030 \pm 0.019$ & $1.427 \pm 0.021$ & 1.228 \\
\hline $\mathrm{MLD}+\mathrm{K}_{1}$ & $3.516 \pm 0.033$ & $2.656 \pm 0.028$ & 3.086 & $5.372 \pm 0.051$ & $5.011 \pm 0.043$ & 5.191 & $1.287 \pm 0.008$ & $1.698 \pm 0.010$ & 1.492 \\
\hline $\mathrm{MLD}+\mathrm{K}_{2}$ & $3.605 \pm 0.043$ & $2.724 \pm 0.040$ & 3.164 & $5.622 \pm 0.038$ & $5.251 \pm 0.032$ & 5.436 & $1.421 \pm 0.011$ & $1.926 \pm 0.014$ & 1.673 \\
\hline SLD & $3.116 \pm 0.029$ & $2.715 \pm 0.026$ & 2.915 & $4.460 \pm 0.043$ & $3.775 \pm 0.038$ & 4.117 & $1.076 \pm 0.016$ & $1.331 \pm 0.020$ & 1.203 \\
\hline $\mathrm{SLD}+\mathrm{K}_{1}$ & $3.575 \pm 0.041$ & $2.981 \pm 0.038$ & 3.278 & $5.441 \pm 0.056$ & $4.449 \pm 0.051$ & 4.945 & $1.377 \pm 0.013$ & $1.610 \pm 0.017$ & 1.493 \\
\hline $\mathrm{SLD}+\mathrm{K}_{2}$ & $3.949 \pm 0.021$ & $3.279 \pm 0.019$ & 3.614 & $6.110 \pm 0.048$ & $4.977 \pm 0.040$ & 5.543 & $1.409 \pm 0.017$ & $1.659 \pm 0.020$ & 1.534 \\
\hline Mean & 4.261 & 3.570 & & 5.707 & 4.811 & & 1.339 & 1.737 & \\
\hline \multirow{7}{*}{$\mathrm{CD}$ at $5 \%$} & \multicolumn{3}{|c|}{ Genotype $(G)=0.047$} & \multicolumn{3}{|c|}{ Genotype $(\mathrm{G})=0.087$} & \multicolumn{3}{|c|}{ Genotype $(\mathrm{G})=0.017$} \\
\hline & \multicolumn{3}{|c|}{ Water treatment $(\mathrm{WT})=0.081$} & \multicolumn{3}{|c|}{ Water treatment $(\mathrm{WT})=0.152$} & \multicolumn{3}{|c|}{ Water treatment $(\mathrm{WT})=0.030$} \\
\hline & \multicolumn{3}{|c|}{$\mathrm{G} \times \mathrm{WT}=0.115$} & \multicolumn{3}{|c|}{$\mathrm{G} \times \mathrm{WT}=0.214$} & \multicolumn{3}{|c|}{$\mathrm{G} \times \mathrm{WT}=0.042$} \\
\hline & \multicolumn{3}{|c|}{$\operatorname{Potassium}(K)=0.047$} & \multicolumn{3}{|c|}{$\operatorname{Potassium}(K)=0.087$} & \multicolumn{3}{|c|}{$\operatorname{Potassium}(\mathrm{K})=0.017$} \\
\hline & \multicolumn{3}{|c|}{$\mathrm{G} \times \mathrm{K}=\mathrm{N} / \mathrm{A}$} & \multicolumn{3}{|c|}{$\mathrm{G} \times \mathrm{K}=\mathrm{N} / \mathrm{A}$} & \multicolumn{3}{|c|}{$\mathrm{G} \times \mathrm{K}=\mathrm{N} / \mathrm{A}$} \\
\hline & \multicolumn{3}{|c|}{$\mathrm{WT} \times \mathrm{K}=0.115$} & \multicolumn{3}{|c|}{$\mathrm{WT} \times \mathrm{K}=0.214$} & \multicolumn{3}{|c|}{$\mathrm{WT} \times \mathrm{K}=0.042$} \\
\hline & \multicolumn{3}{|c|}{$\mathrm{G} \times \mathrm{WT} \times \mathrm{K}=0.163$} & \multicolumn{3}{|c|}{$\mathrm{G} \times \mathrm{WT} \times \mathrm{K}=0.303$} & \multicolumn{3}{|c|}{$\mathrm{G} \times \mathrm{WT} \times \mathrm{K}=0.060$} \\
\hline
\end{tabular}

$F I=$ Fully irrigation, $F D=$ Fully Drought, $M E D=$ Moderate early drought, $S E D=$ Severe early drought, $M L D=$ Moderate late drought, $S L D=$ Severe late drought, $K_{1}=500 \mathrm{ppm}$ ( potassium treatment), $K_{2}=700 \mathrm{ppm}$ (potassium treatment) 


\section{Table 07: Effect of water stress and potassium on CAT activity (UAmg-1 protein) of sweet corn at different growth stages}

\begin{tabular}{|c|c|c|c|c|c|c|c|c|c|}
\hline \multirow{3}{*}{ Treatment } & \multicolumn{9}{|c|}{ Sampling stages } \\
\hline & \multicolumn{3}{|c|}{ Vegetative } & \multicolumn{3}{|c|}{ Silking } & \multicolumn{3}{|c|}{ Post silking } \\
\hline & SUGAR-75 & NSC901B & Mean & SUGAR-75 & NSC901B & Mean & SUGAR-75 & NSC901B & Mean \\
\hline FI & $1.673 \pm 0.018$ & $1.367 \pm 0.011$ & 1.521 & $1.098 \pm 0.048$ & $1.324 \pm 0.031$ & 1.211 & $0.798 \pm 0.010$ & $0.925 \pm 0.008$ & 0.861 \\
\hline $\mathrm{FI}+\mathrm{K}_{1}$ & $2.004 \pm 0.031$ & $1.564 \pm 0.019$ & 1.784 & $1.395 \pm 0.039$ & $1.583 \pm 0.027$ & 1.489 & $0.924 \pm 0.017$ & $1.030 \pm 0.013$ & 0.977 \\
\hline $\mathrm{FI}+\mathrm{K}_{2}$ & $2.120 \pm 0.043$ & $1.753 \pm 0.027$ & 1.936 & $1.493 \pm 0.053$ & $1.834 \pm 0.045$ & 1.663 & $1.034 \pm 0.025$ & $1.159 \pm 0.023$ & 1.096 \\
\hline FD & $2.655 \pm 0.029$ & $2.054 \pm 0.019$ & 2.354 & $1.667 \pm 0.069$ & $1.887 \pm 0.032$ & 1.777 & $1.279 \pm 0.029$ & $1.545 \pm 0.019$ & 1.412 \\
\hline $\mathrm{FD}+\mathrm{K}_{1}$ & $4.009 \pm 0.050$ & $2.915 \pm 0.015$ & 3.462 & $2.583 \pm 0.058$ & $2.830 \pm 0.029$ & 2.706 & $1.816 \pm 0.014$ & $2.070 \pm 0.011$ & 1.943 \\
\hline $\mathrm{FD}+\mathrm{K}_{2}$ & $4.301 \pm 0.087$ & $3.243 \pm 0.027$ & 3.772 & $2.809 \pm 0.049$ & $3.038 \pm 0.037$ & 2.923 & $1.955 \pm 0.023$ & $2.147 \pm 0.017$ & 2.051 \\
\hline MED & $2.204 \pm 0.061$ & $1.890 \pm 0.038$ & 0.227 & $1.940 \pm 0.065$ & $2.164 \pm 0.062$ & 2.052 & $1.090 \pm 0.038$ & $1.334 \pm 0.028$ & 1.212 \\
\hline $\mathrm{MED}+\mathrm{K}_{1}$ & $2.975 \pm 0.013$ & $2.475 \pm 0.008$ & 2.725 & $2.522 \pm 0.038$ & $2.705 \pm 0.021$ & 2.613 & $1.504 \pm 0.029$ & $1.907 \pm 0.017$ & 1.705 \\
\hline $\mathrm{MED}+\mathrm{K}_{2}$ & $3.107 \pm 0.022$ & $2.608 \pm 0.013$ & 2.857 & $2.871 \pm 0.043$ & $3.051 \pm 0.036$ & 2.961 & $1.656 \pm 0.036$ & $2.094 \pm 0.027$ & 1.875 \\
\hline SED & $3.122 \pm 0.039$ & $2.393 \pm 0.029$ & 2.757 & $1.754 \pm 0.026$ & $2.006 \pm 0.021$ & 1.882 & $1.232 \pm 0.059$ & $1.334 \pm 0.039$ & 1.283 \\
\hline $\mathrm{SED}+\mathrm{K}_{1}$ & $4.901 \pm 0.073$ & $3.637 \pm 0.053$ & 4.269 & $2.841 \pm 0.031$ & $3.149 \pm 0.028$ & 2.995 & $1.749 \pm 0.072$ & $1.813 \pm 0.062$ & 1.781 \\
\hline $\mathrm{SED}+\mathrm{K}_{2}$ & $5.276 \pm 0.098$ & $3.852 \pm 0.117$ & 4.564 & $3.069 \pm 0.073$ & $3.450 \pm 0.059$ & 3.259 & $1.823 \pm 0.068$ & $1.880 \pm 0.038$ & 1.851 \\
\hline MLD & $1.876 \pm 0.037$ & $1.523 \pm 0.029$ & 1.699 & $1.471 \pm 0.048$ & $1.834 \pm 0.034$ & 1.652 & $1.034 \pm 0.042$ & $1.154 \pm 0.027$ & 1.094 \\
\hline $\mathrm{MLD}+\mathrm{K}_{1}$ & $2.206 \pm 0.046$ & $1.887 \pm 0.038$ & 2.046 & $1.839 \pm 0.046$ & $2.219 \pm 0.021$ & 2.029 & $1.364 \pm 0.039$ & $1.472 \pm 0.045$ & 1.418 \\
\hline $\mathrm{MLD}+\mathrm{K}_{2}$ & $2.305 \pm 0.053$ & $1.812 \pm 0.049$ & 2.058 & $1.898 \pm 0.027$ & $2.310 \pm 0.019$ & 2.104 & $1.416 \pm 0.032$ & $1.529 \pm 0.011$ & 1.472 \\
\hline SLD & $1.524 \pm 0.032$ & $1.276 \pm 0.011$ & 1.401 & $1.874 \pm 0.062$ & $2.125 \pm 0.010$ & 1.999 & $1.129 \pm 0.052$ & $1.370 \pm 0.027$ & 1.249 \\
\hline $\mathrm{SLD}+\mathrm{K}_{1}$ & $1.901 \pm 0.018$ & $1.537 \pm 0.007$ & 1.719 & $2.454 \pm 0.071$ & $2.635 \pm 0.029$ & 2.544 & $1.366 \pm 0.073$ & $1.635 \pm 0.053$ & 1.500 \\
\hline $\mathrm{SLD}+\mathrm{K}_{2}$ & $1.991 \pm 0.073$ & $1.612 \pm 0.037$ & 1.801 & $2.567 \pm 0.032$ & $2.783 \pm 0.016$ & 2.675 & $1.501 \pm 0.054$ & $1.726 \pm 0.048$ & 1.613 \\
\hline Mean & 2.786 & 2.188 & & 2.117 & 2.384 & & 1.370 & 1.562 & \\
\hline \multirow{7}{*}{$\mathrm{CD}$ at $5 \%$} & \multicolumn{3}{|c|}{$\operatorname{Genotype}(\mathrm{G})=0.036$} & \multicolumn{3}{|c|}{ Genotype $(\mathrm{G})=0.022$} & \multicolumn{3}{|c|}{$\operatorname{Genotype}(G)=0.020$} \\
\hline & \multicolumn{3}{|c|}{ Water treatment $(\mathrm{WT})=0.062$} & \multicolumn{3}{|c|}{ Water treatment $(\mathrm{WT})=0.038$} & \multicolumn{3}{|c|}{ Water treatment $(\mathrm{WT})=0.035$} \\
\hline & \multicolumn{3}{|c|}{$\mathrm{G} \times \mathrm{WT}=0.087$} & \multicolumn{3}{|c|}{$\mathrm{G} \times \mathrm{WT}=0.054$} & \multicolumn{3}{|c|}{$\mathrm{G} \times \mathrm{WT}=0.049$} \\
\hline & \multicolumn{3}{|c|}{$\operatorname{Potassium}(\mathrm{K})=0.036$} & \multicolumn{3}{|c|}{$\operatorname{Potassium}(\mathrm{K})=0.022$} & \multicolumn{3}{|c|}{$\operatorname{Potassium}(\mathrm{K})=0.020$} \\
\hline & \multicolumn{3}{|c|}{$\mathrm{G} \times \mathrm{K}=0.050$} & \multicolumn{3}{|c|}{$\mathrm{G} \times \mathrm{K}=0.031$} & \multicolumn{3}{|c|}{$\mathrm{G} \times \mathrm{K}=\mathrm{N} / \mathrm{A}$} \\
\hline & \multicolumn{3}{|c|}{$\mathrm{WT} \times \mathrm{K}=0.087$} & \multicolumn{3}{|c|}{$\mathrm{WT} \times \mathrm{K}=0.054$} & \multicolumn{3}{|c|}{$\mathrm{WT} \times \mathrm{K}=0.049$} \\
\hline & \multicolumn{3}{|c|}{$\mathrm{G} \times \mathrm{WT} \times \mathrm{K}=0.123$} & \multicolumn{3}{|c|}{$\mathrm{G} \times \mathrm{WT} \times \mathrm{K}=0.076$} & \multicolumn{3}{|c|}{$\mathrm{G} \times \mathrm{WT} \times \mathrm{K}=0.070$} \\
\hline
\end{tabular}

$F I=$ Fully irrigation, $F D=$ Fully Drought, $M E D=$ Moderate early drought, $S E D=$ Severe early drought, MLD= Moderate late drought, $S L D=$ Severe late drought, $K_{1}=500 \mathrm{ppm}$ ( potassium treatment), $K_{2}=700 \mathrm{ppm}$ (potassium treatment) 
Enhanced activity of antioxidant enzymes is associated with defense mechanism of a plant against many biotic and abiotic stresses. Sedghi et al., (2012) observed a strong correlation between antioxidant enzymes and plant tolerance to drought stress. According to Wang et al., (2009) tolerant species have higher antioxidants over susceptible one in stress conditions. Our findings regarding augmentation in antioxidant enzymes in drought stress are in agreement with those of sunflower (Gunes et al., 2008) and cowpea (Manivannan et al., 2007). Detrimental superoxide radical is changed into hydrogen peroxide by SOD and hydrogen peroxide is further broken down into oxygen and hydrogen by other CAT, POD and APX (Ozkur et al., 2009). Activity of all the antioxidative enzymes were augmented in our investigations in water deficit conditions and exogenously applied potassium further increased the antioxidative enzymes. Exogenously applied potassium augmented the SOD, peroxidase and catalase activity in wheat genotype (Wei et al., 2013). Increase in potassium concentration is linked with increased activity of those enzymes which are involved in detoxification of ROS (Cakmak, 2005). Liang et al., (2007), witnessed rise in the activity of superoxide dismutase (SOD), catalase (CAT), and peroxidase (POD) in Zingiber officinale with increasing potassium concentration. Jan et al., (2017) also suggested that activity of POD, SOD and CAT is improved by applying potassium in stressed conditions.

\section{Yield attributes}

The results revealed that both genotypes showed almost equal number of rows in ear irrespective of the stress. Maximum increase in number of rows in ear was found in genotype SUGAR-75 at fully irrigation treatment in response to 700 ppmpotassium concentration (Table 08). Results revealed that ear length was significantly affected by water stress. More reduction in ear length was noticed in NSC901B as compared to SUGAR-75 under stress conditions (Figure- 1-4). However, maximum reduction was observed in fully drought condition in both genotypes. Maximum ear length was observed under fully irrigated stage in both genotypes at $700 \mathrm{ppm}$ potassium concentration. Decrease in the number of ears per plant was observed in both the genotypes. Maximum number of ears per plant was observed in SUGAR-75 as compared to NSC901B (Table 08).

Water stress resulted in marked decrease in the number of kernels per ear in both genotypes. Decrease in the number of kernels per ear was considerably higher in NSC901B than SUGAR-75 (Table 09). Increase in the number of kernels per plant in response to applied potassium was observed in control and revived after stress plants. The genotype SUGAR-75 had significantly higher biomass than NSC901B. Water stress resulted in significant reduction in biomass (158.2 g) in SUGAR -75 and (136.2 g) in NSC901B at fully drought treatment (Table 09). Potassium application resulted in significant increase in biomass in both cultivars under control and stress treatments.

At the present investigation, water deficit caused a significance decrease in rows in ear, biomass of ear, kernels number, ear length and number of ears per plant in both the cultivars. It may be due to the reduction in production of photosynthates that led to the reduction in overall yield (Afzal et al., 2014). It gives a clear indication that drought tolerant genotype showed less reduction in yield in contrast to susceptible ones. The total percentage reduction was more in genotype NSC901B than SUGAR-75. Rivera-Hernandez et al., (2010) stated that decrease in (EL) was due to consequence of drought stress, which leads to decline in photosynthesis and biomass accumulation of kernels. Moser et al., (2006) investigated that drought at initial flowering stage substantially reduced the kernel rows. However, the highest decrement was observed when stress was applied at kernel formation stage (Devi and Kar, 2013). Potassium increased the yield components and hasadiverse role in metabolic processes (Zulkarnain et al., 2009). Kumar et al., (2014) reported that kernel yield was increased significantly by applying potassium. 


\section{Table 08: $\quad$ Effect of water stress and potassium onthe number of rows in ear and ear length $(\mathrm{cm})$ and} number of ears per plant of sweet corn

\begin{tabular}{|c|c|c|c|c|c|c|c|c|c|}
\hline \multirow{2}{*}{ Treatment } & \multicolumn{3}{|c|}{ Number of rows in ear } & \multicolumn{3}{|c|}{ Ear length $(\mathrm{cm})$} & \multicolumn{3}{|c|}{ Number of ears per plant } \\
\hline & SUGAR-75 & NSC901B & Mean & SUGAR-75 & NSC901B & Mean & SUGAR-75 & NSC901B & Mean \\
\hline FI & $12 \pm 0.068$ & $12 \pm 0.038$ & 12 & $21.5 \pm 0.098$ & $19.5 \pm 0.071$ & 20.5 & $2 \pm 0.009$ & $2 \pm 0.008$ & 2 \\
\hline $\mathrm{FI}+\mathrm{K}_{1}$ & $12 \pm 0.056$ & $12 \pm 0.051$ & 12 & $22.0 \pm 0.088$ & $21.3 \pm 0.045$ & 21.65 & $2 \pm 0.007$ & $2 \pm 0.004$ & 2 \\
\hline $\mathrm{FI}+\mathrm{K}_{2}$ & $14 \pm 0.082$ & $12 \pm 0.048$ & 13 & $23.5 \pm 0.069$ & $22.5 \pm 0.048$ & 23.0 & $2 \pm 0.013$ & $2 \pm 0.010$ & 2 \\
\hline FD & $10 \pm 0.118$ & $10 \pm 0.047$ & 10 & $15.5 \pm 0.054$ & $14.6 \pm 0.035$ & 15.05 & $1 \pm 0.015$ & $1 \pm 0.013$ & 1 \\
\hline $\mathrm{FD}+\mathrm{K}_{1}$ & $10 \pm 0.137$ & $12 \pm 0.052$ & 11 & $16.7 \pm 0.038$ & $15.5 \pm 0.087$ & 16.10 & $1 \pm 0.034$ & $1 \pm 0.031$ & 1 \\
\hline $\mathrm{FD}+\mathrm{K}_{2}$ & $12 \pm 0.112$ & $12 \pm 0.032$ & 12 & $18.0 \pm 0.170$ & $17.8 \pm 0.143$ & 17.90 & $1 \pm 0.076$ & $1 \pm 0.065$ & 1 \\
\hline MED & $12 \pm 0.121$ & $12 \pm 0.037$ & 12 & $17.1 \pm 0.156$ & $16.3 \pm 0.133$ & 16.71 & $2 \pm 0.023$ & $1 \pm 0.021$ & 1.5 \\
\hline $\mathrm{MED}+\mathrm{K}_{1}$ & $12 \pm 0.110$ & $12 \pm 0.017$ & 12 & $17.5 \pm 0.176$ & $17.0 \pm 0.165$ & 17.25 & $2 \pm 0.032$ & $1 \pm 0.028$ & 1.5 \\
\hline $\mathrm{MED}+\mathrm{K}_{2}$ & $12 \pm 0.132$ & $12 \pm 0.027$ & 12 & $19.0 \pm 0.157$ & $19.1 \pm 0.144$ & 19.05 & $1 \pm 0.017$ & $1 \pm 0.011$ & 1 \\
\hline SED & $10 \pm 0.154$ & $10 \pm 0.084$ & 10 & $16.5 \pm 0.241$ & $15.0 \pm 0.211$ & 15.75 & $1 \pm 0.064$ & $1 \pm 0.054$ & 1 \\
\hline $\mathrm{SED}+\mathrm{K}_{1}$ & $10 \pm 0.136$ & $10 \pm 0.065$ & 10 & $18.1 \pm 0.211$ & $15.1 \pm 0.181$ & 16.60 & $1 \pm 0.031$ & $1 \pm 0.028$ & 1 \\
\hline $\mathrm{SED}+\mathrm{K}_{2}$ & $12 \pm 0.067$ & $12 \pm 0.048$ & 12 & $20.0 \pm 0.197$ & $17.0 \pm 0.163$ & 18.5 & $1 \pm 0.018$ & $1 \pm 0.015$ & 1 \\
\hline MLD & $12 \pm 0.128$ & $12 \pm 0.032$ & 12 & $18.5 \pm 0.143$ & $17.2 \pm 0.120$ & 17.85 & $2 \pm 0.064$ & $1 \pm 0.058$ & 1.5 \\
\hline $\mathrm{MLD}+\mathrm{K}_{1}$ & $12 \pm 0.129$ & $12 \pm 0.029$ & 12 & $19.6 \pm 0.340$ & $17.0 \pm 0.312$ & 18.3 & $2 \pm 0.006$ & $1 \pm 0.005$ & 1.5 \\
\hline $\mathrm{MLD}+\mathrm{K}_{2}$ & $12 \pm 0.097$ & $14 \pm 0.038$ & 13 & $20.0 \pm 0.166$ & $18.5 \pm 0.129$ & 29.25 & $2 \pm 0.017$ & $1 \pm 0.015$ & 1.5 \\
\hline SLD & $10 \pm 0.098$ & $10 \pm 0.231$ & 10 & $16.2 \pm 0.120$ & $14.5 \pm 0.122$ & 15.35 & $1 \pm 0.011$ & $2 \pm 0.008$ & 1.5 \\
\hline $\mathrm{SLD}+\mathrm{K}_{1}$ & $10 \pm 0.125$ & $10 \pm 0.015$ & 10 & $18.1 \pm 0.174$ & $16.0 \pm 0.153$ & 17.05 & $1 \pm 0.032$ & $2 \pm 0.029$ & 1.5 \\
\hline $\mathrm{SLD}+\mathrm{K}_{2}$ & $12 \pm 0.132$ & $12 \pm 0.023$ & 12 & $21.5 \pm 0.168$ & $17.0 \pm 0.154$ & 19.25 & $2 \pm 0.019$ & $2 \pm 0.015$ & 2 \\
\hline Mean & 11.44 & 11.55 & & 18.85 & 17.27 & & 1.5 & 1.3 & \\
\hline \multirow{7}{*}{$\mathrm{CD}$ at $5 \%$} & \multicolumn{3}{|c|}{$\operatorname{Genotype}(\mathrm{G})=0.138$} & \multicolumn{3}{|c|}{ Genotype $(\mathrm{G})=\mathrm{N} / \mathrm{A}$} & \multicolumn{3}{|c|}{$\operatorname{Genotype}(G)=0.018$} \\
\hline & \multicolumn{3}{|c|}{ Water treatment $(\mathrm{WT})=0.238$} & \multicolumn{3}{|c|}{ Water treatment $(\mathrm{WT})=0.342$} & \multicolumn{3}{|c|}{ Water treatment $(\mathrm{WT})=0.031$} \\
\hline & \multicolumn{3}{|c|}{$\mathrm{G} \times \mathrm{WT}=0.337$} & \multicolumn{3}{|c|}{$\mathrm{G} \times \mathrm{WT}=0.484$} & \multicolumn{3}{|c|}{$\mathrm{G} \times \mathrm{WT}=0.043$} \\
\hline & \multicolumn{3}{|c|}{$\operatorname{Potassium}(\mathrm{K})=0.138$} & \multicolumn{3}{|c|}{$\operatorname{Potassium}(K)=0.198$} & \multicolumn{3}{|c|}{$\operatorname{Potassium}(\mathrm{K})=0.018$} \\
\hline & \multicolumn{3}{|c|}{$\mathrm{G} \times \mathrm{K}=\mathrm{N} / \mathrm{A}$} & \multicolumn{3}{|c|}{$\mathrm{G} \times \mathrm{K}=0.279$} & \multicolumn{3}{|c|}{$\mathrm{G} \times \mathrm{K}=\mathrm{N} / \mathrm{A}$} \\
\hline & \multicolumn{3}{|c|}{$\mathrm{WT} \times \mathrm{K}=0.337$} & \multicolumn{3}{|c|}{$\mathrm{WT} \times \mathrm{K}=0.484$} & \multicolumn{3}{|c|}{$\mathrm{WT} \times \mathrm{K}=0.043$} \\
\hline & \multicolumn{3}{|c|}{$\mathrm{G} \times \mathrm{WT} \times \mathrm{K}=0.477$} & \multicolumn{3}{|c|}{$\mathrm{G} \times \mathrm{WT} \times \mathrm{K}=0.685$} & \multicolumn{3}{|c|}{$\mathrm{G} \times \mathrm{WT} \times \mathrm{K}=0.062$} \\
\hline
\end{tabular}

$F I=$ Fully irrigation, $F D=$ Fully Drought, $M E D=$ Moderate early drought, $S E D=$ Severe early drought, MLD= Moderate late drought, $S L D=$ Severe late drought, $K_{1}=500 \mathrm{ppm}$ ( potassium treatment), $K_{2}=700 \mathrm{ppm}$ (potassium treatment)

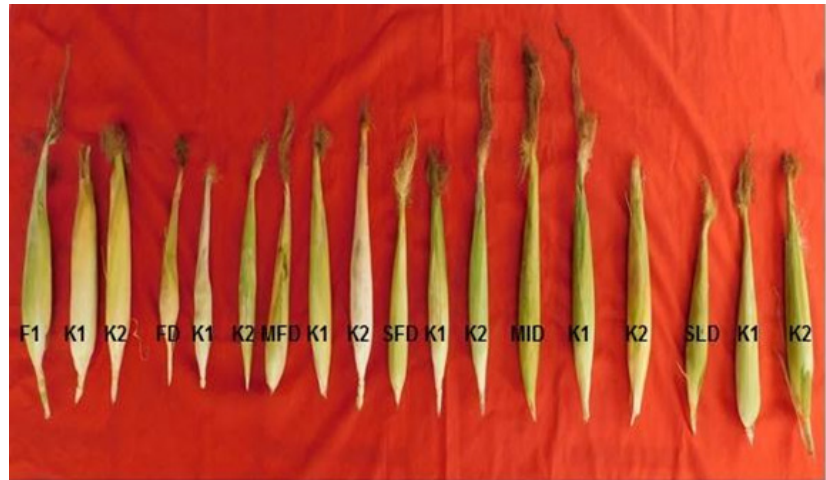

Figure 01: Showing cob of sweet corn genotype SUGAR-75 with husk under different treatment of drought and potassium.

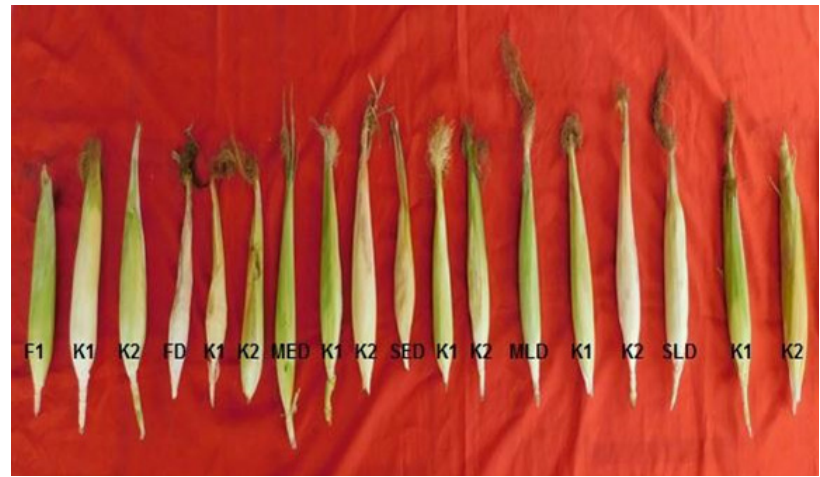

Figure 02: Showing cob of sweet corn genotype NSC901B with husk under different treatment of drought and potassium 


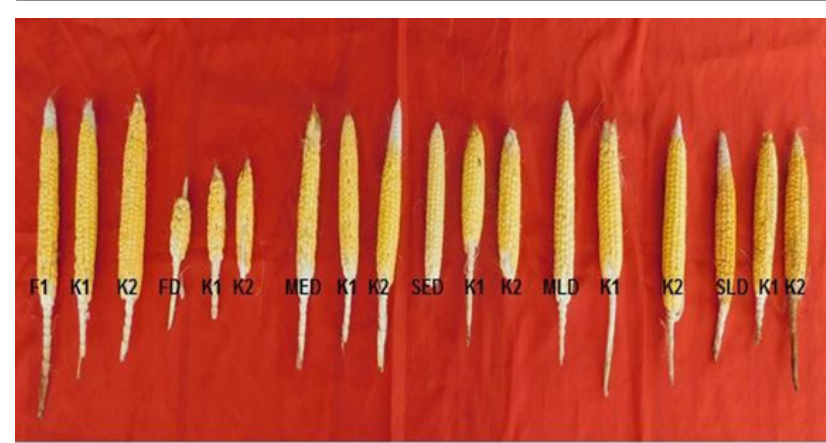

Figure 03: Showing cob of sweet corn genotype SUGAR-75 without husk under different treatment of drought and potassium

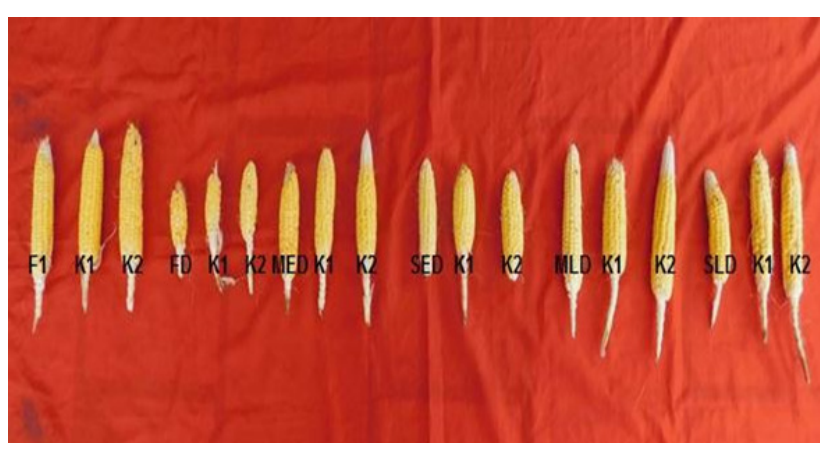

Figure 04: Showing cob of sweet corn genotype NSC901B without husk under different treatment of drought and potassium

Table 09: $\quad$ Effect of water stress and potassium on the number of kernels per ear and biomass of ear (g) of sweet corn

\begin{tabular}{|c|c|c|c|c|c|c|}
\hline \multirow{2}{*}{ Treatment } & \multicolumn{3}{|c|}{ Number of kernels per ear } & \multicolumn{3}{|c|}{ Biomass of ear $(\mathrm{g})$} \\
\hline & SUGAR-75 & NSC901B & Mean & SUGAR-75 & NSC901B & Mean \\
\hline FI & $540 \pm 2.367$ & $510 \pm 2.112$ & 525 & $230.5 \pm 1.053$ & $205.8 \pm 1.003$ & 218.1 \\
\hline $\mathrm{FI}+\mathrm{K}_{1}$ & $560 \pm 1.100$ & $526 \pm 1.001$ & 543 & $255.7 \pm 1.823$ & $213.3 \pm 1.432$ & 234.5 \\
\hline $\mathrm{FI}+\mathrm{K}_{2}$ & $626 \pm 1.798$ & $538 \pm 1.321$ & 582 & $280.4 \pm 2.579$ & $217.5 \pm 2.154$ & 248.9 \\
\hline FD & $280 \pm 2.332$ & $284 \pm 2.109$ & 282 & $158.2 \pm 1.096$ & $136.2 \pm 1.005$ & 147.2 \\
\hline $\mathrm{FD}+\mathrm{K}_{1}$ & $312 \pm 1.348$ & $288 \pm 1.206$ & 300 & $188.1 \pm 1.489$ & $143.3 \pm 1.212$ & 165.7 \\
\hline $\mathrm{FD}+\mathrm{K}_{2}$ & $340 \pm 1.472$ & $308 \pm 1.123$ & 324 & $195.3 \pm 2.312$ & $149.4 \pm 2.103$ & 172.3 \\
\hline MED & $410 \pm 1.200$ & $378 \pm 1.098$ & 394 & $181.3 \pm 3.647$ & $175.6 \pm 2.876$ & 178.4 \\
\hline $\mathrm{MED}+\mathrm{K}_{1}$ & $436 \pm 3.225$ & $388 \pm 2.345$ & 412 & $187.8 \pm 0.744$ & $182.7 \pm 0.543$ & 185.2 \\
\hline $\mathrm{MED}+\mathrm{K}_{2}$ & $456 \pm 2.654$ & $402 \pm 2.432$ & 429 & $198.2 \pm 1.289$ & $188.9 \pm 1.076$ & 193.5 \\
\hline SED & $368 \pm 1.687$ & $328 \pm 1.265$ & 348 & $168.5 \pm 1.811$ & $153.7 \pm 1.432$ & 161.1 \\
\hline $\mathrm{SED}+\mathrm{K}_{1}$ & $380 \pm 1.611$ & $336 \pm 1.321$ & 358 & $177.2 \pm 0.744$ & $164.4 \pm 0.654$ & 170.8 \\
\hline $\mathrm{SED}+\mathrm{K}_{2}$ & $386 \pm 1.200$ & $346 \pm 1.103$ & 366 & $184.9 \pm 1.012$ & $172.2 \pm 1.002$ & 178.5 \\
\hline MLD & $468 \pm 1.627$ & $438 \pm 1.324$ & 453 & $196.3 \pm 1.829$ & $189.5 \pm 1.436$ & 192.9 \\
\hline $\mathrm{MLD}+\mathrm{K}_{1}$ & $488 \pm 1.432$ & $442 \pm 1.212$ & 465 & $205.8 \pm 2.514$ & $203.2 \pm 2.143$ & 204.5 \\
\hline $\mathrm{MLD}+\mathrm{K}_{2}$ & $504 \pm 1.542$ & $454 \pm 1.243$ & 479 & $210.2 \pm 1.543$ & $208.4 \pm 1.324$ & 209.3 \\
\hline SLD & $410 \pm 1.376$ & $372 \pm 1.276$ & 391 & $174.4 \pm 1.232$ & $180.3 \pm 1.103$ & 177.3 \\
\hline $\mathrm{SLD}+\mathrm{K}_{1}$ & $428 \pm 1.221$ & $378 \pm 1.198$ & 403 & $182.4 \pm 1.453$ & $186.5 \pm 1.245$ & 184.4 \\
\hline $\mathrm{SLD}+\mathrm{K}_{2}$ & $442 \pm 1.453$ & $388 \pm 1.234$ & 415 & $195.4 \pm 1.287$ & $196.2 \pm 1.043$ & 195.8 \\
\hline Mean & 435.2 & 394.6 & & 198.3 & 181.5 & \\
\hline \multirow{7}{*}{$\mathrm{CD}$ at $5 \%$} & \multicolumn{3}{|c|}{ Genotype $(\mathrm{G})=4.760$} & \multicolumn{3}{|c|}{ Genotype $(\mathrm{G})=2.117$} \\
\hline & \multicolumn{3}{|c|}{ Water treatment $(\mathrm{WT})=8.245$} & \multicolumn{3}{|c|}{ Water treatment $(\mathrm{WT})=3.667$} \\
\hline & \multicolumn{3}{|c|}{$\mathrm{G} \times \mathrm{WT}=11.661$} & \multicolumn{3}{|c|}{$\mathrm{G} \times \mathrm{WT}=5.186$} \\
\hline & \multicolumn{3}{|c|}{$\operatorname{Potassium}(\mathrm{K})=4.760$} & \multicolumn{3}{|c|}{$\operatorname{Potassium}(\mathrm{K})=2.117$} \\
\hline & \multicolumn{3}{|c|}{$\mathrm{G} \times \mathrm{K}=6.732$} & \multicolumn{3}{|c|}{$\mathrm{G} \times \mathrm{K}=\mathrm{N} / \mathrm{A}$} \\
\hline & \multicolumn{3}{|c|}{$\mathrm{WT} \times \mathrm{K}=11.66$} & \multicolumn{3}{|c|}{$\mathrm{WT} \times \mathrm{K}=5.186$} \\
\hline & \multicolumn{3}{|c|}{$\mathrm{G} \times \mathrm{WT} \times \mathrm{K}=16.49$} & \multicolumn{3}{|c|}{$\mathrm{G} \times \mathrm{WT} \times \mathrm{K}=7.334$} \\
\hline
\end{tabular}

$F I=$ Fully irrigation, $F D=$ Fully Drought, $M E D=$ Moderate early drought, $S E D=$ Severe early drought, MLD= Moderate late drought , $S L D=$ Severe late drought, $K_{1}=500 \mathrm{ppm}$ ( potassium treatment) , $K_{2}=700 \mathrm{ppm}$ (potassium treatment) 


\section{CONCLUSIONS}

It was evident from the results that water deficit at any critical crop growth stage severely affected water relations, antioxidative defense systemand yield attributes of sweet corn. Soil applied potassium in drought stressed plants improved the water relations, activity of antioxidative enzymes and yield attributes. We conclude that var. sugar75 of sweet corn combined with adequate external potassium supply may be a promising strategy for better growth in water deficit conditions.

\section{ACKNOWLEDGEMENT}

Authors are highly thankful IARI, New Delhi, India for providing certified seeds of sweet corn (Zea mays convar. Saccharata, var. rugosa) and Department of Botany, Kurukshetra University, Kurukshetra, India for providing the necessary facilities for conducting the experimental work.

\section{REFERENCES}

Aebi, H. (1983). Catalase In: Methods of enzymatic analysis (Ed. H.U. Bergmeyer). Verlag Chemie. 2: 673-684. ISBN: 978-0-12-091302-2.

Afkari, B.A. Qasimov, N. and Yarnia, M. (2009). Effects of drought stress and potassium on some of the physiological and morphological traits of sunflower (Helianthus annuus L.) cultivars. Journal of Applied Botany and Food Quality.7: 448-451. ISSN: 1459-0255.

Afzal, A. Gulzar, I. Shahbaz, M. and Ashraf, M. (2014). Water deficit-induced regulation of growth, gas exchange, chlorophyll fluorescence, inorganic nutrient accumulation and antioxidative defense mechanism in mungbean [Vigna radiata (L.) Wilczek]. Journal of Applied Botany and Food Quality.87:147-156. https://doi.org/10.5073/JABFQ.2014.087.022.

Ahmad, Z. Anjum, S. Waraich, E.A. Ayub, M.A. Ahmad, T. Tariq, R.M.S. Ahmad R. and Iqbal. M.A. (2018). Growth, physiology, and biochemical activities of plant responses with foliar potassium application under drought stress - a review. Journal of Plant Nutrition. 41(13): 1734-1743. https://doi.org/10.1080/01904167.2018.1459688.

Ashraf, M.A. Akbar, A. Askari, S.H. Iqbal, M. Rasheed, R. and Hussain, I. (2018). Recent advances in abiotic stress tolerance of plants through chemical priming: an overview. in advances in seed priming. Springer: Berlin/Heidelberg, Germany, pp. 51-79. https://doi.org/10.24203/AJAFS. V8I1.6068.

Ashraf, R. Fayyaz-ul, H. Ahmed, M. and Shabbir, G. (2017). Wheat physiological response under drought. In: Ahmed, M. and Stockle, C.O. (Eds.), quantification of climate variability, adaptation and mitigation for agricultural sustainability. Springer International Publishing, ChamSwitzerland. pp. 211-231.

Cakmak, I. (2005). The role of potassium in alleviating detrimental effects of abiotic stresses in plants. Journal of Plant Nutrition and Soil Science, Oldenburg. 168(4): 521-530. https://doi. org/10.1002/jpln.200420485.

Clavel, D. Drame, N.K. Roy-Macauley, H.Braconnier, S. and Laffray, D. (2005). Analysis of early responses to drought associated with field drought adaptation in four sahelian groundnut (Arachis hypogaea L.) cultivars. Environmental and Experimental Botany.54: 219-230. https:// doi.org/10.1016/j.envexpbot.2004.07.008. 
Devi, S.H. and Kar, M. (2013). Amelioration of moisture stress effect by $\mathrm{CaCl}_{2}$ pre-treatment in upland rice. Indian Journal of Plant Physiology.18: 384-387.https://doi.org/10.1007/s40502014-0058-y.

Dewanto, V. Wu, X. and Liu, R.H. (2002). Processed sweet corn has higher antioxidant activity. Journal of Agricultural and Food Chemistry.14: 50(17). https://doi.org/10.1021/jf0255937.

FAO. (2019). World Food and Agriculture Statistical Year book. https://doi.org/10.4060/cb4477en.

Giannopolitis, C.N. and Ries, S.K. (1977). Superoxide dismutase I: occurrence in higher plants. Plant Physiology.59:309-314. https://doi.org/10.1104/pp.59.2.309.

Gunes, A. Pilbeam, D. Inal, A. and Coban, S. (2008): Influence of silicon on sunflower cultivars under drought stress: Growth, antioxidant mechanisms and lipid peroxidation. Communications in SoilScience and Plant Analysis.39:1885-1903.https://doi.org/10.1080/0103620802134651.

Jain, M. Kataria, S. Hirve, M. and Prajapati, R. (2019). Water deficit stress effects and responses in maize. Plant Abiotic Stress Tolerance.pp. 129-151. https://doi.org/10.1007/978-3-030-06118$0 \_5$.

Jan, A.U. Hadi, F. Nawaz, M.A. and Rahman, K. (2017). Potassium and zinc increase tolerance to salt stress in wheat (Triticum aestivum L.). Plant Physiology and Biochemistry.116: 139-149. https://doi.org/10.1016/j.plaphy.2017.05.008.

Janda, T. Szalai, G. Giauffret, C.Paldi, E. and Ducruet, J.M. (1999). The thermoluminescence "after glow" band as a sensitive indicator of abiotic stresses in plants. Zeitschriftfür Naturforschung. 54: 629- 633. https://doi.org/10.1515/znc-1999-9-1003.

Junpatiw, A. Lertrat, K.Lomthaisong, K. and Tangwongchai, R. (2013). Effects of steaming, boiling and frozen storage on carotenoid contents of various sweet corn cultivars. International Food Research Journal. 20(5): 2219-2225. ISSN: 22317546.

Kumar, P. Kumar, P. Singh, T. Singh, A.K. and Yadav, R. (2014). Effect of different potassium levels on mungbean under custard apple based agri-horti system. African Journal of Agricultural Research. 9: 728-734. https://doi.org/10.5897/AJAR2013.7883.

Liang, T.B. Wang, Z.L. Wang, R.J. Liu, L.L. and Shi, C.Y. (2007). Effects of potassium humate on ginger root growth and its active oxygen metabolism. The journal of applied ecology.18: 813817. ISSN: 1001-9332.

Lucier, G. and Dettmann R.L. (2008).Vegetables and melons situation and outlookyearbook. Washington DC. United States Department of Agriculture.

Manivannan, P. Jaleel, C.A. Kishorekumar, A. Sankar, B. Somasundaram, R. Sridharan, R. and Panneerselvam, R. (2007). Changes in antioxidant metabolism of Vigna unguiculata (L.) Walp. by propiconazole under water deficit stress. Colloids Surf B Biointerfaces. 57: 69-74. doi: 10.1016/j.colsurfb.2007.01.004.

Moser, S.B. Feil, B. Jampatong, S. and Stamp, P. (2006). Effects of pre-anthesis drought, nitrogen fertilizer rate, and variety on grain yield, yield components, and harvest index of tropical maize. Agriculture Water Management. 81: 41-58. https://doi.org/10.1016/j.agwat.2005.04.005.

Ozkur, O. Ozdemir, F. Bor, M. and Turkan, I. 2009. Physiochemical and antioxidant responses of the perennial xerophyte Capparis ovata desf. to drought. Environmental Experimental Botany. 66: 487-492. https://doi.org/10.1016/j.envexpbot.2009.04.003. 
Putter, J. (1974). Peroxidase. Weinham Verlag Chemie.

Ranum, P. Pẽna-Rosas, J.P. and Garcia-Casal, M.N. (2014). Global maize production, utilization, and consumption. Annals of the New York Academy of Sciences. 1312: 105-112.https://doi. org/10.1111/nyas.12396.

Raza, M.A.S. Saleem, M.F. Shah, G.M. Khan, I.H. and Raza, A. (2014). Exogenous application of glycine betaine and potassium for improving water relations and grain yield of wheat under drought. Journal of Soil Science and Plant Nutrition. 14(2): 348-364. http://dx.doi.org/10.4067/ S0718-95162014005000028.

Richardson, K.J. Lewis, K.H. Krishnamurthy, P.K. Kent, C. Wiltshire, A.J. and Hanlon, H.M. (2018). Food security outcomes under a changing climate: Impacts of mitigation and adaptaion on vulnerablity to food insecurity. Climatic Change. 147:327-341. https://doi.org/10.1007/s10584018-2137-y.

Rivera-Hernandez, B. Carrillo-Avila, E. Obrador-Olan, J.J. Juarez-Lopez, J.F. and Aceves-Navarro, L.A. (2010). Morphological quality of sweet corn (Zea mays L.) ears as response to soil moisture tension and phosphate fertilization in Campeche, Mexico. Agricultural water management. 97(9): 1365-1374. https://doi.org/10.1016/j.agwat.2010.04.001.

Sedghi, M. Sharifi, R.S.Pirzad, A.R. and Balaneji, B.A. (2012). Phytohormonal regulation of antioxidant systems in petals of drought stressed pot marigold (Calendula officinalis L.). Journal of Agriculture, Science and Technology.14: 869-878. eISSN: 1561-7645.

Serraj, R. and Sinclair, T.R. (2002). Osmolyte accumulation: can it really help increase crop yield under drought conditions? Plant, Cell \& Environment.25: 333-341. https://doi.org/10.1046/ j.1365-3040.2002.00754.x.

Sheoran, O.P. Tonk, D.S. Kaushik, L.S. Hasija, R.C. and Pannu, R.S. (1998). Statistical Software Package for Agricultural Research Workers. Recent Advances in information theory, Statistics \& Computer Applications by Hooda D.S. and Hasija R.C. Department of Mathematics Statistics, CCS HAU, Hisar. 139-143.

United States Department of Agriculture, Agricultural Research Service (2019). National Nutrient Database for Standard Reference Release.

Wang, W.B. Kim, Y.H. Lee, H.S. Kim, K.Y. Deng, X.P. and Kwak, S.S. (2009). Analysis of antioxidant enzyme activity during germination of alfalfa under salt and drought stress. Plant Physiology and Biochemistry. 47: 570-577. https://doi.org/10.1016/j.plaphy.2009.02.009.

Waraich, E.A. Ahmad, R. Saifullah, M. Ashraf, Y. and Ehsanullah, E. (2011). Role of mineral nutrition in alleviation of drought stress in plants. Australian Journal of Crop Science. 5: 764-777. ISSN18352693.

Weatherley, P.E. (1950). Studies in the water relations of the cotton plant. The field measurement of water deficits in leaves. New Phytologist. 49: 81-87. https://doi.org/10.1111/j.1469-8137.1950. tb05146.x.

Wei, J. Li, C. Li, Y. Jiang, G. Cheng, G. and Zheng, Y. (2013). Effects of external potassium (K) supply on drought tolerances of two contrasting winter wheat cultivars. PLoS ONE. 8(7): e69737. https://doi.org/10.1371/journal.pone.0069737. 
Zhang, L.X. Gao, M.Shengxiu, L.I. Alva, K.A. and Ashraf, M. (2014). Potassium fertilization mitigates the adverse effects of drought on selected Zea mays cultivars. Turkish Journal of Botany.38: 713-723. https://doi.org/10.3906/bot-1308-47.

Zhang, L.X. Gao, M. Zhang, L.S. Li, B.Z. Han, M.Y. Alva, K.A. and Ashraf, M. (2013). Role of exogenous glycinebetaine and humic acid in mitigating drought stress-induced adverse effects in Malus robusta seedlings. Turkish Journal of Botany.37: 920-929. https://doi.org/10.3906/ bot-1212-21.

Zhang, L.X. Li, S.X. and Liang, Z.S. (2009). Differential plant growth and osmotic effects of two maize (Zea mays L.) cultivars to exogenous glycine betaine application under drought stress. Plant Growth Regulation.58: 297-305. https://doi.org/10.1007/s10725-009-9379-7.

Zulkarnain, W.M. Ismail, M.R. Ashrafuzzaman, M. Saud, H.M. and Haroun, I.C. (2009). Rice growth and yield under rain shelter house as influenced by different water regimes. International Journal of Agriculture and Biology.11: 566-570. https://doi.org/09-010/PFZ/2009/11-5-566-570 